\title{
QUIVER W-ALGEBRAS
}

\author{
TARO KIMURA AND VASILY PESTUN
}

\begin{abstract}
For a quiver with weighted arrows we define gauge-theory K-theoretic Walgebra generalizing the definition of Shiraishi et al., and Frenkel and Reshetikhin. In particular, we show that the $q q$-character construction of gauge theory presented by Nekrasov is isomorphic to the definition of the $\mathrm{W}$-algebra in the operator formalism as a commutant of screening charges in the free field representation. Besides, we allow arbitrary quiver and expect interesting applications to representation theory of generalized Borcherds-Kac-Moody Lie algebras, their quantum affinizations and associated W-algebras.
\end{abstract}

\section{Contents}

1. Introduction

Acknowledgements

2. Quiver gauge theory

2.1. Quiver

2.2. Cartan matrix and Kac-Moody algebra

2.3. Quiver sheaves

2.4. Moduli space

2.5. Universal sheaf

2.6. Equivariant version

2.7. Partition function

2.8. Fundamental matter

2.9. Local observables

2.10. Extended partition function

2.11. Localization

3. Quiver gauge theory on $\mathbb{C}^{2}$

3.1. Automorphism group $G L(\mathbf{Q})$

3.2. Automorphism groups $G L(\mathbf{N})$ and $G L(\mathbf{M})$

3.3. Complete group of equivariance

3.4. Fundamental matter as background of higher times

3.5. Localization in quiver theory on $\mathbb{C}^{2}$

3.6. Cotangent moduli space

3.7. Two commutative reductions

3.8. Quantum $q$-geometric Langlands Duality

3.9. Intermediate reduction

3.10. The set of eigenvalues

3.11. The partition function

3.12. Reflection of the index

3.13. The ordered partition function

3.14. Extended partition function is a state 
3.15. Free bosons and vertex operators $\quad 12$

3.16. Screening charges $\quad 12$

3.17. The Ward identities 13

3.18. The Y-operators $\quad 13$

3.19. The OPE of $Y$ and $S \quad 14$

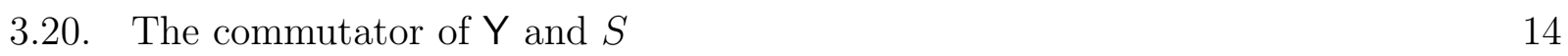

3.21. The V-operators $\quad 15$

4. W-algebra $\quad 15$

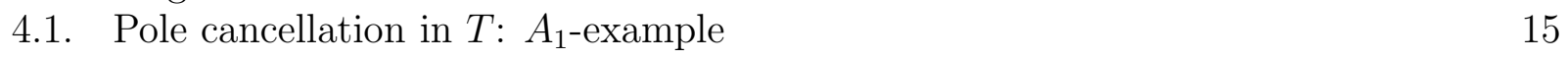

4.2. Commutator of $T$ and $S$ vanishing: $A_{1}$-example $\quad 16$

4.3. W-algebra of $A_{1}$-quiver $\quad 17$

4.4. W-algebra of quiver: definition $\quad 17$

5. Examples $\quad 17$

5.1. Commutator of $T$ and $S$ vanishing: general quiver, local reflection $\quad 18$

5.2. Higher weight currents 19

5.3. Higher weight current in the $A_{1}$ example $\quad 19$

5.4. Degeneration and derivatives 2

5.5. Edge loop: $\widehat{A}_{0}$-example $\quad 21$

5.6. W-algebra of hyperbolic quiver example $\quad 22$

6. Applications $\quad 23$

6.1. Toda scaling limit $\quad 23$.

6.2. Affine type 23

6.3. Nahm transform $\quad 24$

References $\quad 24$

\section{INTRODUCTION}

Let $\Gamma$ be a quiver with $\mu$-weighted arrows $\mu: \Gamma_{1} \rightarrow \mathbb{C}^{\times}$. We construct two-parametric algebra $W_{q_{1}, q_{2}}(\Gamma)$ from the equivariant K-theory on the moduli space of $\Gamma$-quiver sheaves on $\mathbb{C}_{q_{1}, q_{2}}^{2}$. If a quiver is simply-laced Dynkin graph, our construction agrees with FrenkelReshetikhin's definition of $W_{q_{1}, q_{2}}\left(\mathfrak{g}_{\Gamma}\right)$ [1] on the one hand. On the other hand, it explains that certain observables of the gauge theory, coming from the $q_{2}$-lift of the gauge-theory construction of $q_{1}$-characters in [2], described in details and called $q_{1} q_{2}$-characters in [3] , can be promoted to operator valued currents that form non-commutative associative current algebra $W_{q_{1}, q_{2}}\left(\mathfrak{g}_{\Gamma}\right)$.

In our constructions the $q_{1} q_{2}$-character currents are valued in the algebra of differential operators in higher times $t_{i, 1}, \ldots, t_{i, \infty}$ of the gauge theory [4]. We show that the algebra of these differential operators is equivalent to the Heisenberg algebra of $q_{1} q_{2}$-bosons used by Shiraishi et al. [5] and Frenkel-Reshetikhin [1, 6] to define $W_{q_{1}, q_{2}}\left(\mathfrak{g}_{\Gamma}\right)$ algebra. Specializing to $\mathfrak{g}_{\Gamma}$ of ADE type (in this case $\Gamma$ has no loops and hence $\mu$-parameters represent necessarily the trivial class in $H_{1}\left(\Gamma, \mathbb{C}^{\times}\right)$and hence are gauged away) we show that the pole cancellation construction of [2] and [3] developed from the cut cancellation construction of [7], is isomorphic to the definition of $W_{q_{1}, q_{2}}\left(\mathfrak{g}_{\Gamma}\right)$ algebra in [1] as the commutant of screening charges, 
hence explaining the isomorphism between the gauge theory construction presented in [3] and the algebraic contruction of [1].

The gauge theory definition of $W_{q_{1}, q_{2}}(\Gamma)$ algebra is symmetric in exchange $q_{1} \leftrightarrow q_{2}$. However, for the free field realization there is a choice between $\left(q_{1}, q_{2}\right)$ and $\left(q_{2}, q_{1}\right)$. The equivalence between the two realizations, transparent from the gauge theory, leads to 'quantum $q$-geometric' Langlands equivalence. The duality of W-algebras and the connection with geometric Langlands was first found in [8]. The 'quantum q-geometric' Langlands [1, 9] degenerates to the (CFT) 'quantum geometric' Langlands duality $\beta \leftrightarrow \beta^{-1}$ in the limit $q_{1}=e^{\epsilon_{1}}, q_{2}=e^{\epsilon_{2}}$ with $\epsilon_{1}, \epsilon_{2} \rightarrow 0$ and $\beta=-\epsilon_{1} / \epsilon_{2}$, and further down in the limit $\epsilon_{1}=\hbar, \epsilon_{2}=0$ to the 'geometric' Langlands duality [10-12]. For a survey of duality of W-algebras and its connection with the geometric Langlands program see [1] and [13] section 8.6.

In the language of complex integrable systems, and in the reverse order, the 'geometric' Langlands duality $\left(\epsilon_{1}=\hbar, \epsilon_{2}=0\right)$ is T-duality along the fibers of the phase space of Hitchin integrable system. The K-theory lift to $q_{1}=e^{\hbar}, q_{2}=1$ converts Hitchin integrable system on flat curve $C$ to group valued Hitchin integrable system on $C$ [14], equivalently to the integrable system of periodic monopoles on $C \times S^{1}$ [7]. In the limit $q_{2}=1$ there is isomorphism, found in [15], between the algebra $W_{q_{1}, q_{2}}\left(\mathfrak{g}_{\Gamma}\right)$, which turns into commutative algebra, and the K-theory ring of the category of certain representations of the quantum loop group $\mathbf{U}_{q_{1}}\left(L \mathfrak{g}_{\Gamma}\right)$

$$
W_{q_{1}, 1}\left(\mathfrak{g}_{\Gamma}\right) \simeq K\left(\operatorname{Rep} \mathbf{U}_{q_{1}}\left(L \mathfrak{g}_{\Gamma}\right)\right)
$$

The character of the R-matrix maps the elements of $W_{q_{1}, 1}\left(\mathfrak{g}_{\Gamma}\right)$ to the commuting Hamiltonians of quantum integrable system [2, 15, 16].

The geometric realization of $\mathbf{U}_{q_{1}}\left(L \mathfrak{g}_{\Gamma}\right)$ and of $q_{1}$-characters in $W_{a_{1}, 1}\left(\mathfrak{g}_{\Gamma}\right)$ was obtained by Nakajima [17] after Ringel [18], Lusztig [19], Ginzburg-Vasserot [20] from the equivariant K-theory of the $\mathbb{C}_{q_{1}}^{\times}$-equivariant cotangent bundle of the moduli space $T_{q_{1}}^{*} \mathfrak{M}(\Gamma, \mathbb{C M o d})$ of $\Gamma$ quiver representations in the category of vector spaces $\mathbb{C}$-Mod. To see the $q_{2}$-parameter one needs to consider the central extension of $\mathbf{U}_{q_{1}}\left(L \mathfrak{g}_{\Gamma}\right)$ to $\mathbf{U}_{q_{1}}\left(\widehat{\mathfrak{g}}_{\Gamma}\right)$ (quantum Drinfeld affinization of $\left.\mathfrak{g}_{\Gamma}\right)$. The central extension is missing in Nakajima's construction which concerns only the specialization of $\mathbf{U}_{q_{1}}\left(\widehat{\mathfrak{g}}_{\Gamma}\right)$ by the trivial center to $\mathbf{U}_{q_{1}}\left(L \mathfrak{g}_{\Gamma}\right)[1$

Compared to Nakajima, we replace a point by a complex variety $\mathcal{S}$ and replace $\mathbb{C}$ Mod by $\mathcal{O}_{\mathcal{S}} \operatorname{Mod}$, so that we consider equivariant K-theory on the moduli space $\mathfrak{M}\left(\Gamma, \mathcal{O}_{\mathcal{S}} \mathbf{M o d}\right)$ of $\Gamma$-quiver representations in the category of coherent sheaves on a complex variety $\mathcal{S}$. For $\mathcal{S}=\mathbb{C}_{q_{1}, q_{2}}^{2}$ we recover $W_{q_{1}, q_{2}}\left(\mathfrak{g}_{\Gamma}\right)$ from K-theory on $\mathfrak{M}\left(\Gamma, \mathcal{O}_{\mathbb{C}_{q_{1}, q_{2}}}\right.$ Mod $)$. As proposed by Nekrasov in [3] using complex 4-dimensional setup this should be equivalent to considering the K-theory on the $q_{2}$-twisted fiber-parity-inversed total space of the tangent bundle to Nakajima's quiver variety $\Pi T_{q_{2}} T_{q_{1} q_{2}}^{*} \mathfrak{M}(\Gamma, \mathbb{C M o d})$.

We expect that $\mathrm{K}$-theory definition of quiver $\mathrm{W}$-algebra $W\left(\Gamma, \mathbb{C}_{q_{1}, q_{2}}^{2}\right)$ can be given in a more geometric sense in the more general situation when $\mathbb{C}_{q_{1}, q_{2}}^{2}$ is replaced by a generic complex variety $\mathcal{S}$ factorized into a product $\mathcal{S}=\mathcal{S}_{1} \times \mathcal{S}_{2}$ and we expect that the duality $\mathcal{S}_{1} \leftrightarrow \mathcal{S}_{2}$ will be lifted to higher Langlands duality. The relation to cohomological Hall algebra of quiver [22] remains to be clarified.

\footnotetext{
$1_{\text {The parameters }}\left(q_{1}^{\frac{1}{2}}, q_{2}^{-\frac{1}{2}}\right)$ are called by $(q, t)$ in [1, 5, 9]. However, the parameter $t$ in Nakajima's $(q, t)$-characters [21], which grades the cohomological degree, has different meaning from the present $q_{2}$.
} 
This paper takes equivariant K-theory as example of generalized cohomological theory corresponding to the supersymmetric 5 d theory reduced on $S^{1}$. However, all constructions remain intact if equivariant $\mathrm{K}$-theory is replaced by the ordinary equivariant cohomology ( $4 \mathrm{~d}$ theory) or by equivariant elliptic cohomology (6d theory reduced on elliptic curve). Consequently, the geometric construction of K-theoretic W-algebra can be scaled to its Yangian version [1, 15, 16, 23] using cohomology and lifted to the elliptic version [24, 25] using elliptic cohomology.

For $A_{r}$-quivers the defining relation of the present note between gauge theory and $W\left(A_{r}\right)$ algebra after the $90^{\circ}$ brane rotation (the exchange between the rank of the gauge group in the quiver nodes and the rank of the quiver [26], equivalently Nahm transform, fiber-base duality, S-duality) implies the AGT duality of [27 29]. The invariance under the brane rotation of the gauge theory partition function is clear from the formalism of refined topological vertex 30 32 and was explicitly checked in 33. The relation between quiver gauge theories and $\mathrm{W}$-algebras in terms of Toda conformal blocks for finite ADE quivers also appeared in [34]. Also it would be interesting to interpret the higher times and the meaning of the presented $W(\Gamma)$-symmetry in the context of topological string on toric Calabi-Yau realization of the gauge theory partition function for ADE and affine ADE quivers [35].

We do not restrict $\Gamma$ to be quiver of finite Dynkin type and consequently expect interesting applications to representation theory of (quantum affinization of) generalized BorcherdsKac-Moody Lie algebras, such as $E_{11}$ symmetry prominently appearing in M-theory or Borcherds Monster Lie algebra for Conway-Norton moonshine. The affine and hyperbolic quivers generate new $\mathrm{W}$-algebras describing affine (such as sinh-Gordon) and hyperbolic quantum 2d Toda models.

Notes. The origins of the $q$-Virasoro symmetry the case of single node quiver $\Gamma=A_{1}=\circ$ to $\operatorname{Vir}_{q_{1}, q_{2}}=W_{q_{1}, q_{2}}\left(A_{1}\right)$ algebra can be traced to Eynard's $q$-deformed single matrix model [3638]. Elliptic version of matrix model is discussed in [39]. It would be interesting to explore $\Gamma$-quiver matrix models beyond Dynkin graphs of finite and affine type [40].

The regularity of $q q$-characters was explained by Nekrasov in the talk in Strings 2014 and multiple other talks. In [41] the regularity for a linear quiver was interpreted in the language of the quantum toroidal algebra $\mathbf{U}_{q_{1}, q_{2}}\left(\widehat{\widehat{\mathfrak{g l}}}_{1}\right)$ which by Nakajima's construction [42 48] acts on the instanton moduli spaces on $\mathbb{C}_{q_{1}, q_{2}}^{2}$ for each individual node.

Acknowledgements. We thank for discussions and comments Alexey Sevastyanov, Edward Frenkel, Nikita Nekrasov and Samson Shatashvili. TK is grateful to Institut des Hautes Études Scientifiques for hospitality where a part of this work has been done. The work of TK was supported in part by Keio Gijuku Academic Development Funds, JSPS Grantin-Aid for Scientific Research (Nos. JP13J04302 and JP17K18090), the MEXT-Supported Program for the Strategic Research Foundation at Private Universities "Topological Science" (No. S1511006), JSPS Grant-in-Aid for Scientific Research on Innovative Areas "Topological Materials Science" (No. JP15H05855), and "Discrete Geometric Analysis for Materials Design" (No. JP17H06462). VP acknowledges grant RFBR 15-01-04217 and RFBR 16-0201021. The research of VP on this project has received funding from the European Research Council (ERC) under the European Union's Horizon 2020 research and innovation program (QUASIFT grant agreement 677368). 


\section{Quiver Gauge THEORY}

In this section we define the extended partition function of quiver gauge theories [4, 49, 50].

2.1. Quiver. Let $\Gamma$ be a quiver with the set of nodes $\Gamma_{0}$ and the set of arrows $\Gamma_{1}$. By $i, j \in \Gamma_{0}$ we label the nodes, and by $e: i \rightarrow j$ we denote an arrow $e$ from the source $i=s(e)$ to the target $j=t(e)$. We allow loops and multiple arrows.

2.2. Cartan matrix and Kac-Moody algebra. A quiver $\Gamma$ defines $\left|\Gamma_{0}\right| \times\left|\Gamma_{0}\right|$ matrix $\left(c_{i j}\right)_{i, j \in \Gamma_{0}}$

$$
c_{i j}=2-\#(e: i \rightarrow j)-\#(e: j \rightarrow i)
$$

that is called quiver Cartan matrix. By definition, the quiver Cartan matrix $c$ is symmetric. If there are no single node loops, all diagonal entries of the quiver Cartan matrix are equal to 2 and such Cartan matrix defines Kac-Moody algebra $\mathfrak{g}_{\Gamma}$ with Dynkin graph $\Gamma$.

2.3. Quiver sheaves. Choose the space-time to be a complex variety $\mathcal{S}$ with structure sheaf $\mathcal{O}_{\mathcal{S}}$, and let $\operatorname{Coh}(\mathcal{S})$ denote the category of coherent sheaves on $\mathcal{S}$ (the category $\mathcal{O}_{\mathcal{S}}$ Mod of $\mathcal{O}_{\mathcal{S}}$-modules). Let $\operatorname{Coh}(\mathcal{S})_{\Gamma}=\operatorname{Rep}(\Gamma, \operatorname{Coh}(\mathcal{S}))$ be the category of representations of quiver $\Gamma$ in $\operatorname{Coh}(\mathcal{S})$. We call $\operatorname{Rep}(\Gamma, \operatorname{Coh}(\mathcal{S}))$ by $\Gamma$-quiver gauge theory on $\mathcal{S}$ : each node $i$ is sent to a sheaf $\mathcal{Y}_{i}$ on $\mathcal{S}$ and each arrow $e: i \rightarrow j$ is sent to an element of $\operatorname{Hom}_{\mathcal{O}_{\mathcal{S}}}\left(\mathcal{Y}_{i}, \mathcal{Y}_{j}\right)$.

In the context of $\mathcal{N}=2$ gauge theories, a sheaf $\mathcal{Y}_{i}$ represents gauge connection in the $i$-th vector multiplet, and an element in $\operatorname{Hom}_{\mathcal{O}_{\mathcal{S}}}\left(\mathcal{Y}_{i}, \mathcal{Y}_{j}\right)$ represents field in the $i \rightarrow j$ bifundamental hypermultiplet.

\subsection{Moduli space. Let}

$$
\mathfrak{M}(\Gamma, \mathcal{S})=\operatorname{Coh}(\mathcal{S})_{\Gamma} / \operatorname{Aut}\left(\operatorname{Coh}(\mathcal{S})_{\Gamma}\right)
$$

be the moduli space of $\Gamma$-quiver sheaves on $\mathcal{S}$. Let $\gamma=\operatorname{ch} \mathcal{Y}$ denote the Chern character of the collection $\mathcal{Y}=\left(\mathcal{Y}_{i}\right)_{i \in \Gamma_{0}}$ so that $\gamma=\left(\gamma_{i}\right)_{i \in \Gamma_{0}}$ with $\gamma_{i}=\operatorname{ch} \mathcal{Y}_{i} \in H^{\bullet}(\mathcal{S})$. The Chern character $\gamma_{i}$ characterizes the topological class of sheaf $\mathcal{Y}_{i}$.

The total moduli space $\mathfrak{M}(\Gamma, \mathcal{S})$ of $\Gamma$-quiver sheaves on $\mathcal{S}$ is a disjoint union over topological sectors

$$
\mathfrak{M}(\Gamma, \mathcal{S})=\coprod_{\gamma} \mathfrak{M}(\Gamma, \mathcal{S})_{\gamma}
$$

Algebraically, the moduli space $\mathfrak{M}(\Gamma, \mathcal{S})$ is a derived stack with virtual tangent bundle $T \mathfrak{M}(\Gamma, \mathcal{S})$ at $\mathcal{Y}$ given by

$$
T_{\mathcal{Y}} \mathfrak{M}(\Gamma, \mathcal{S})=\operatorname{Coh}(\mathcal{S})_{\Gamma}(\mathcal{Y}, \mathcal{Y})[1]
$$

More explicitly,

$$
T_{\mathcal{Y}} \mathfrak{M}(\Gamma, \mathcal{S})^{\bullet}=\bigoplus_{(i \stackrel{e}{\rightarrow} j) \in \Gamma_{1}} \operatorname{Ext}_{\mathcal{O}_{\mathcal{S}}}^{\bullet}\left(\mathcal{Y}_{i}, \mathcal{Y}_{j}\right) \oplus \bigoplus_{i \in \Gamma_{0}} \operatorname{Ext}_{\mathcal{O}_{\mathcal{S}}^{+1}}^{+1}\left(\mathcal{Y}_{i}, \mathcal{Y}_{i}\right)
$$

2.5. Universal sheaf. Let $\mathcal{Y}=\left(\hat{\mathcal{Y}}_{i}\right)_{i \in \Gamma_{0}}$ denote the universal sheaf over $\mathfrak{M}(\Gamma, \mathcal{S}) \times \mathcal{S}$ that is associated to the family of sheaves $\mathcal{Y}_{i}$ on $\mathcal{S}$ parametrized by $\mathfrak{M}(\Gamma, \mathcal{S})$. 
2.6. Equivariant version. Suppose we are given an equivariant action of a complex group $\mathrm{T}$ on the sheaves $\operatorname{Coh}(\mathcal{S})$. Then quiver gauge theory can be defined T-equivariantly. In particular, group $\mathrm{T}$ acts on the moduli space $\mathfrak{M}(\Gamma, \mathcal{S})$ of $\mathrm{T}$-equivariant $\Gamma$-quiver sheaves on $\mathcal{S}$.

2.7. Partition function. Define partition function $Z_{\mathrm{T}}(\Gamma, \mathcal{S})_{\gamma}$ in topological sector $\gamma$ be the T-equivariant index (holomorphic equivariant Euler characteristic) of the structure sheaf on the moduli space of $\Gamma$-quiver sheaves on $\mathcal{S}$ of charge $\gamma$

$$
Z_{\mathrm{T}}(\Gamma, \mathcal{S})_{\gamma}=\sum_{n \in \mathbb{Z}}(-1)^{n} \operatorname{ch}_{\mathrm{T}} H^{n}\left(\mathfrak{M}(\Gamma, \mathcal{S})_{\gamma}, \mathcal{O}_{\mathfrak{M}(\Gamma, \mathcal{S})_{\gamma}}\right)
$$

The total partition function is the sum over the charges

$$
Z_{\mathrm{T}}(\Gamma, \mathcal{S})=\sum_{\gamma} \mathfrak{q}^{\gamma} Z_{\mathrm{T}}(\Gamma, \mathcal{S})_{\gamma}
$$

This partition function in the context of $\mathcal{N}=2$ gauge theories is known under the name K-theoretic Nekrasov partition function, or the partition function of the $5 \mathrm{~d}$ quiver gauge theory reduced on $S^{1}$ [50] where $\mathfrak{q}$ is a union of $\mathfrak{q}_{i}=\exp \left(2 \pi \imath \tau_{i}\right)$ with the complexified coupling constant $\left(\tau_{i}\right)_{i \in \Gamma_{0}}$.

We can write the partition function using the notation of the derived pushforward $\pi_{!}=$ $\sum(-1)^{i} R_{i} \pi_{*}$ for the projection (integration) map $\pi: \mathfrak{M}(\Gamma, \mathcal{S}) \rightarrow$ point

$$
Z_{\mathrm{T}}(\Gamma, \mathcal{S})=\operatorname{ch}_{\mathrm{\top}} \pi_{!} \mathfrak{q}^{\gamma}
$$

By definition, the partition function $Z_{\mathrm{T}, \gamma}$, being a character of a virtual representation in $\operatorname{Rep}(\mathrm{T})$, can be evaluated on an element $\mathrm{t} \in \mathrm{T}$. In the context of Nekrasov's partition function the element $\mathrm{t}$ comprises all equivariant parameters.

2.8. Fundamental matter. Since quiver $\Gamma$ is arbitrary, unlike [2, 7] where $\Gamma$ was of finite or affine type, in the present formalism (anti) fundamental matter for a node $i$ is treated simply as bi-fundamental arrow between the node $i$ and another frozen node, denoted by $i^{\prime}$, which is represented in constant sheaves with gauge coupling constant $\mathfrak{q}_{i^{\prime}}$ turned off.

2.9. Local observables. Let $o \in \mathcal{S}$ be a $\mathrm{T}$-invariant point on space-time $\mathcal{S}$ and let $i_{o}: o \rightarrow \mathcal{S}$ be the inclusion map that naturally induces $i_{o}: \mathfrak{M}(\Gamma, \mathcal{S}) \rightarrow \mathfrak{M}(\Gamma, \mathcal{S}) \times \mathcal{S}$.

We define observable sheaves $\left(\mathbf{Y}_{i}\right)_{i \in \Gamma_{0}}$ over the moduli space $\mathfrak{M}(\Gamma, \mathcal{S})$ as the pullback of the universal sheaf $\left(\hat{\mathcal{Y}}_{i}\right)_{i \in \Gamma_{0}}$ from $\mathfrak{M}(\Gamma, \mathcal{S}) \times \mathcal{S}$ to $\mathfrak{M}(\Gamma, \mathcal{S})$ by the inclusion $i_{o}$

$$
\mathbf{Y}_{i}=i_{o}^{*} \hat{\mathcal{Y}}_{i}
$$

Let $\mathbf{Y}_{i}^{[p]}$ be the $p$-th Adams operation applied to $\mathbf{Y}_{i}$, which is realized using the complex scalar in the vector multiplet as $\operatorname{Tr} \Phi_{i}^{p}$ in $4 \mathrm{~d}$. The sheaves $\left(\mathbf{Y}_{i}^{[p]}\right)_{i \in \Gamma_{0}, p \in \mathbb{Z}_{\geq 1}}$ generate the ring of observables (using the direct sum modulo equivalence from exact sequences as the addition and the tensor product as the multiplication) which is a subring in the T-equivariant Ktheory of sheaves on $\mathfrak{M}(\Gamma, \mathcal{S})$. 
2.10. Extended partition function. We fix a quiver $\Gamma$ and the space-time $\mathcal{S}$ and drop the symbols from the notations.

Associated to the local observables $\left(\mathbf{Y}^{[p]}\right)_{i \in \Gamma_{0}, p \in \mathbb{Z}_{>1}}$, introduce parameters, called higher times $t=\left(t_{i, p}\right)_{i \in \Gamma_{0}, p \in \mathbb{Z}_{\geq 1}}$ and Chern-Simons levels $\left(\kappa_{i}\right)_{i \in \Gamma_{0}}$. We treat higher times $t_{i, p}$ as the conjugate variable to $\mathbf{Y}_{i}^{[p]}$ in the sense of the generating function [4]

$$
Z_{\mathrm{T}}(t)=\operatorname{ch}_{\mathrm{T}} \pi_{!} \mathfrak{q}^{\gamma} \prod_{i \in \Gamma_{0}}\left[\operatorname{det} \hat{\mathbf{Y}}_{i}\right]^{\kappa_{i}} \exp \left(\sum_{p=1}^{\infty} t_{i, p} \mathbf{Y}_{i}^{[p]}\right)
$$

2.11. Localization. Suppose that space of the T-fixed points in $\mathfrak{M}$ is a discrete set of points $\mathfrak{M}^{\top}$ with inclusion $i_{\mathcal{T}}: \mathfrak{M}^{\top} \hookrightarrow \mathfrak{M}$. Then the generating function (2.10) can be computed by localization formuld:

$$
Z_{\mathbf{T}}(t)=\sum_{\mathfrak{M}^{\top}} \mathfrak{q}^{\gamma} \exp \left(\sum_{p=1}^{\infty} \frac{1}{p} \operatorname{ch}_{\mathbf{T}}\left(T_{\mathfrak{M}^{\top}}^{*} \mathfrak{M}\right)^{[p]}\right) \prod_{i \in \Gamma_{0}} \operatorname{ch}_{\mathbf{T}}\left[\operatorname{det} i_{\mathrm{T}}^{*} \hat{\mathbf{Y}}\right]^{\kappa_{i}} \exp \left(\sum_{p=1}^{\infty} t_{i, p} \operatorname{ch}_{\mathbf{T}}\left(i_{\mathrm{T}}^{*} \mathbf{Y}_{i}\right)^{[p]}\right)
$$

\section{Quiver Gauge theOry ON $\mathbb{C}^{2}$}

In this section we specialize to the space-time $\mathcal{S}=\mathbb{C}^{2}$ with marked point $o \in \mathcal{S}$ and the natural action of complex group $G L(2)$ on $\mathcal{S}$ by with fixed point $o$.

3.1. Automorphism group $G L(\mathbf{Q})$. We denote by $\mathbf{Q}$ the fiber of the cotangent bundle to $\mathcal{S}$ at $O$

$$
\mathrm{Q}=T_{o}^{*} \mathcal{S}
$$

Then $\mathbf{Q}$ is the defining module for the group of its automorphisms $G L(\mathbf{Q})$. We split $\mathbf{Q}=$ $\mathbf{Q}_{1} \oplus \mathbf{Q}_{2}$ with respect to Cartan torus

$$
\mathrm{T}_{\mathbf{Q}} \simeq G L\left(\mathbf{Q}_{1}\right) \times G L\left(\mathbf{Q}_{2}\right) \subset G L(\mathbf{Q})
$$

and define $q_{1}, q_{2}$ to be corresponding characters

$$
q_{1}=\operatorname{ch} \mathbf{Q}_{1}, \quad q_{2}=\operatorname{ch} \mathbf{Q}_{2}, \quad Q=\operatorname{ch}_{\mathrm{T}_{\mathbf{Q}}} \mathbf{Q}=q_{1}+q_{2}, \quad q=q_{1} q_{2}=\operatorname{ch} \Lambda^{2} \mathbf{Q}
$$

Remark. The parameters $\left(q_{1}, q_{2}\right)$ are exponentiated (multiplicative) $\epsilon$-parameters of the gauge theory [7, 51].

3.2. Automorphism groups $G L(\mathbf{N})$ and $G L(\mathbf{M})$. Let $\mathcal{O}(\mathcal{S})=\mathbb{C}\left[z_{1}, z_{2}\right]$, the ring of polynomials in two variables, be the coordinate ring of $\mathcal{S}=\mathbb{C}^{2}$. The space of sections of a coherent sheaf on $\mathcal{S}=\mathbb{C}^{2}$ is a $\mathcal{O}(\mathcal{S})$-module. Then $\Gamma$-quiver gauge theory on $\mathcal{S}$ is identified with representation of $\Gamma$ in $\mathcal{O}(\mathcal{S})$-modules. We can take $\mathbb{C}^{2} \simeq \mathbb{P}^{2} \backslash \mathbb{P}_{\infty}^{1}$ and fix framing at the $\mathbb{P}_{\infty}^{1}$.

For a $\Gamma$-quiver sheaf $\mathcal{Y}$, let $n \in \mathbb{Z}_{>0}^{\Gamma_{0}}$ denote the rank and let $k \in \mathbb{Z}_{\geq 0}^{\Gamma_{0}}$ denote the instanton charge

$$
\mathrm{n}(\mathcal{Y})=\operatorname{ch}_{0} \mathcal{Y}, \quad \mathrm{k}(\mathcal{Y})=-\operatorname{ch}_{2} \mathcal{Y}
$$

Let $\mathbf{N}=\left(\mathbf{N}_{i}\right)_{i \in \Gamma_{0}}$ be the framing space associated to $\Gamma_{0}$ part of quiver (nodes). To each node $i$ we associate the framing $\mathbf{N}_{i} \simeq \mathbb{C}^{\mathbf{n}_{i}}$ for the respective sheaf $\mathcal{Y}_{i}$ on $\mathcal{S}$. Let $G L(\mathbf{N})=$

\footnotetext{
${ }^{2}$ Lefshetz - Grothendieck-Hirzebruch-Riemann-Roch-Atiyah-Singer formula
} 
$\prod_{i \in \Gamma_{0}} G L\left(\mathbf{N}_{i}\right)$ be the respective group of automorphisms and let $\mathbf{T}_{\mathbf{N}}$ be a Cartan torus of $G L(\mathbf{N})$. Let $N_{i}$ be the character of $\mathbf{N}_{i}$

$$
N_{i}=\operatorname{ch}_{\mathbf{T}_{\mathbf{N}}} \mathbf{N}_{i}=\nu_{i, 1}+\cdots+\nu_{i, \mathbf{n}_{i}}
$$

Remark. The parameters $\left(\nu_{i, \alpha}\right)_{i \in \Gamma_{0}, \alpha \in\left[1 \ldots n_{i}\right]}$ are the multiplicative Coulomb parameters of the gauge theory.

Let $\mathbf{M}=\left(\mathbf{M}_{e}\right)_{e \in \Gamma_{1}}$ be the framing space associated to $\Gamma_{1}$ part of quiver (arrows). To each individual arrow $e$ we associate one-dimensional mass-twisting space $\mathbf{M}_{e} \simeq \mathbb{C}$. Let $G L(\mathbf{M})=\prod_{e \in \Gamma_{1}} G L\left(\mathbf{M}_{e}\right)$ be the respective group of automorphisms and let $\mathbf{T}_{\mathbf{M}}$ be a Cartan torus in $G L(\mathbf{M})$. Let $M$ be the character of $\mathbf{M}$

$$
M=\operatorname{ch}_{\mathrm{T}_{\mathbf{M}}} \mathbf{M}=\mu
$$

Remark. The parameters $\left(\mu_{e}\right)_{e \in \Gamma_{1}}$ are multiplicative mass parameters of the bifundamental fields $e: i \rightarrow j$ of the gauge theory.

If we assign a multiplicity $\mathrm{m}_{e}$ to an arrow $e \in \Gamma_{1}$, then the mass-twisting space is $\mathbf{M}_{e} \simeq \mathbb{C}^{\mathrm{m}_{e}}$ with the character

$$
M=\operatorname{ch}_{\mathrm{T}_{\mathrm{M}}} \mathrm{M}=\mu_{1}+\cdots+\mu_{\mathrm{m}_{e}}
$$

Since in our formalism $G L\left(\mathbf{M}_{e}\right)$ is reduced to its Cartan torus $\mathbf{T}_{\mathbf{M}_{e}}=\left(\mathbb{C}^{\times}\right)^{\mathrm{m}_{e}}$, the formalism where an arrow $e: i \rightarrow j$ is assigned a multiplicity $\mathrm{m}_{e}$ is equivalent to the formalism where this arrow is replaced by $\mathrm{m}_{e}$ for individual arrows $i \rightarrow j$.

3.3. Complete group of equivariance. For $\Gamma$-quiver gauge theory on $\mathcal{S}=\mathbb{C}^{2}$ we denote by

$$
\mathrm{T}=\mathrm{T}_{\mathbf{Q}} \times \mathrm{T}_{\mathbf{N}} \times \mathrm{T}_{\mathbf{M}}
$$

the Cartan torus in the automorphism group of the moduli space $\mathfrak{M}(\mathcal{S}, \Gamma)$.

3.4. Fundamental matter as background of higher times. Alternatively, fundamental matter can be realized as a background in higher times. To add a fundamental hypermultiplet with multiplicative mass $\mu \in \mathbb{C}^{\times}$to the node $i$ it is sufficient to additively modify the times to

$$
t_{i, p} \rightarrow t_{i, p}+\frac{1}{p} \frac{q^{p}}{\left(1-q_{1}^{p}\right)\left(1-q_{2}^{p}\right)} \mu^{-p}
$$

To simplify presentation we don't keep track of the fundamental matter since it is a particular case of the higher times theory. In the operator formalism, on the other hand, this shift is imposed by additional vertex operators introduced in Sec. 3.21.

3.5. Localization in quiver theory on $\mathbb{C}^{2}$. The localization formula (2.11) to the Tfixed point set $\mathfrak{M}^{\top}$ in the moduli space of $\Gamma$-quiver sheaves on $\mathcal{S}=\mathbb{C}^{2}$ can be explicitly computed [50 52].

The T-fixed sheaves split into the direct sum of one-dimensional T-fixed ideal sheaves, which are classified as $\mathrm{T}_{\mathrm{Q}}$-fixed ideals in $\mathcal{O}(\mathcal{S}) \simeq \mathbb{C}\left[z_{1}, z_{2}\right]$ where the fixed point $o \in \mathbb{C}^{2}$ is the origin $o=(0,0)$. A $\mathrm{T}_{\mathrm{Q}}$-fixed ideal in $\mathbb{C}\left[z_{1}, z_{2}\right]$ of $\mathrm{ch}_{2}=-\mathrm{k}$ is labelled by a partition $(\lambda)=\lambda_{1} \geq \lambda_{2} \geq \cdots \geq 0 \geq 0 \ldots$ of total size $|\lambda|=\sum_{i=1}^{\infty} \lambda_{i}=\mathrm{k}$. Each box $s=\left(s_{1}, s_{2}\right)$ in the partition $\lambda$ with $s_{1} \in[1 \ldots \infty]$ and $s_{2} \in\left[1 \ldots \lambda_{s_{1}}\right]$ is associated to the monomial $z_{1}^{s_{1}-1} z_{2}^{s_{2}-1}$. 
The ideal $\mathbf{I}_{\lambda} \subset \mathcal{O}(\mathcal{S})=\mathbf{I}_{\emptyset}$ is $\mathcal{O}(\mathcal{S})$-generated by all monomials outside of the partition $\lambda$. Let $\mathbf{K}_{\lambda}=\mathbf{I}_{\emptyset} / \mathbf{I}_{\lambda}$ be generated by the monomials in the partition $\lambda$.

A $\mathrm{T}_{\mathbf{N}} \times \mathrm{T}_{\mathrm{Q}}$-fixed $\mathcal{O}(\mathcal{S})$-module $\mathbf{Y}_{\mathcal{S}}=\hat{\mathcal{Y}}(\mathcal{S})$ of rank $\mathrm{n}$ splits into direct sum of $\mathrm{T}_{\mathrm{Q}^{-}}$fixed ideals

$$
\mathbf{Y}_{\mathcal{S}}=\bigoplus_{\alpha \in[1 \ldots \mathrm{n}]} \mathbf{I}_{\lambda_{\alpha}} \otimes \mathbf{N}_{\alpha}
$$

and let $\mathbf{Y} \equiv \mathbf{Y}_{o}=i_{o}^{*} \mathbf{Y}_{\mathcal{S}}$. Then we have in K-theory by localization to $i_{o}: o \hookrightarrow \mathcal{S}$

$$
\left[\mathbf{Y}_{\mathcal{S}}\right]=\left[\mathbf{Y}_{o}\right] /[\Lambda \mathbf{Q}]
$$

where $\Lambda \mathbf{Q}=\sum_{i}(-1)^{i} \Lambda^{i} \mathbf{Q}$ and the division is in formal series. Then

$$
\left[\mathbf{Y}_{o}\right]=[\mathbf{N}]-[\Lambda \mathbf{Q}][\mathbf{K}]
$$

3.6. Cotangent moduli space. From $(2.5)$ we find the K-theory class $\left[T_{\mathcal{Y}}^{*} \mathfrak{M}\right]$ at T-fixed point $\hat{\mathbf{Y}} \in \mathfrak{M}^{\top}$

$$
\left[T_{\hat{\mathbf{Y}}}^{*} \mathfrak{M}\right]=\frac{1}{\left[\Lambda \mathbf{Q}^{\vee}\right]}\left(\sum_{(i \rightarrow j) \in \Gamma_{1}}\left[\mathbf{M}_{e}^{\vee}\right]\left[\mathbf{Y}_{o}\right]_{i}\left[\mathbf{Y}_{o}^{\vee}\right]_{j}-\sum_{i \in \Gamma_{0}}\left[\mathbf{Y}_{o}\right]_{i}\left[\mathbf{Y}_{o}^{\vee}\right]_{i}\right)
$$

3.7. Two commutative reductions. Since the space-time $\mathcal{S}$ is a product $\mathcal{S}=\mathcal{S}_{1} \times \mathcal{S}_{2}$ the reduction from $\mathbf{Y}_{\mathcal{S}}$ to $\mathbf{Y}_{o}$ can be done in two steps in two ways, either first project along $\mathcal{S}_{2}$ and then along $\mathcal{S}_{1}$ (left path) or first project along $\mathcal{S}_{1}$ and then along $\mathcal{S}_{2}$ (right path)

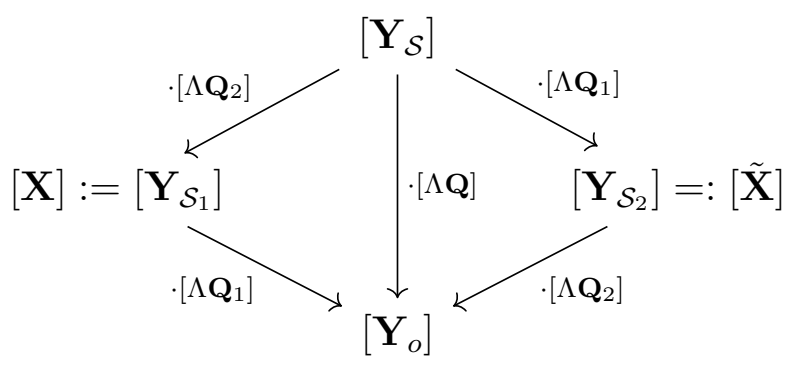

so that it holds

$$
\left[\mathbf{Y}_{o}\right]=\left[\Lambda \mathbf{Q}_{1}\right][\mathbf{X}], \quad\left[\mathbf{Y}_{o}\right]=\left[\Lambda \mathbf{Q}_{2}\right][\tilde{\mathbf{X}}]
$$

Remark. Swapping $\mathcal{S}_{1} \leftrightarrow \mathcal{S}_{2}$ leads to transposition of the partition $\left(\lambda_{i, \alpha}\right) \leftrightarrow\left(\lambda_{i, \alpha}^{\mathrm{T}}\right)$ characterizing the $\mathrm{T}$-fixed point in the moduli space.

3.8. Quantum q-geometric Langlands Duality. The exchange $\mathcal{S}_{1} \leftrightarrow \mathcal{S}_{2}$ in the above diagram leads to the quantum q-geometric Langlands duality $q_{1} \leftrightarrow q_{2}$. See section 1 for references.

3.9. Intermediate reduction. The class $\left[T_{\hat{\mathbf{Y}}}^{*} \mathfrak{M}\right]$ at fixed point $\hat{\mathbf{Y}} \in \mathfrak{M}^{\top}$ in the equation (3.13) of the partition function can be expressed in terms of $[\mathbf{X}] \equiv\left[\mathbf{Y}_{\mathcal{S}_{1}}\right]$

$$
\left[T_{\mathbf{Y}}^{*} \mathfrak{M}\right]=\frac{\left[\Lambda \mathbf{Q}_{1}\right]}{\left[\Lambda \mathbf{Q}_{2}^{\vee}\right]}\left(-\sum_{(i, j) \in \Gamma_{0} \times \Gamma_{0}}[\mathbf{X}]_{i} c_{i j}^{+}[\mathbf{X}]_{j}^{\vee}\right)
$$


and the K-theory valued half Cartan matrix $c_{i j}^{+}$defined as

$$
\left[\mathbf{c}_{i j}^{+}\right]:=\delta_{i j}-\sum_{e: i \rightarrow j}\left[\mathbf{M}_{e}^{\vee}\right]
$$

with Chern character

$$
\operatorname{ch}\left[\mathbf{c}_{i j}^{+}\right]=\delta_{i j}-\sum_{e: i \rightarrow j} \mu_{e}^{-1}
$$

3.10. The set of eigenvalues. Then the Chern characters $X=\operatorname{ch} \mathbf{X}$ at T-fixed point $\lambda$ can be explicitly described. Let

$$
\mathcal{X}_{i}=\left\{x_{i, \alpha, s_{1}}\right\}_{\alpha \in\left[1 \ldots n_{i}\right], s_{1} \in[1 \ldots \infty]}, \quad \mathcal{X}=\bigsqcup_{i \in \Gamma_{0}} \mathcal{X}_{i}
$$

be the set of characters of the monomials associated to boxes $\left(s_{1}, \lambda_{s_{1}}+1\right)$ that generate $\left(\mathbf{Y}_{\mathcal{S}}\right)$ as $\mathcal{O}\left(\mathcal{S}_{2}\right)$-module so that

$$
x_{i, \alpha, s_{1}}=\nu_{i, \alpha} q_{1}^{s_{1}-1} q_{2}^{\lambda_{i, \alpha, s_{1}}}
$$

and $X_{i}=\sum_{x \in \mathcal{X}_{i}} x$. Let $\mathrm{i}: \mathcal{X} \rightarrow \Gamma_{0}$ be the node label so that $\mathrm{i}(x)=i$ for $x \in \mathcal{X}_{i}$.

3.11. The partition function. In terms of $x$-variables the extended partition function (2.11) is

$$
\begin{aligned}
& Z_{\mathrm{T}}(t)=\sum_{\mathcal{X} \in \mathfrak{M}^{\top}} \exp \left(-\sum_{\left(x_{L}, x_{R}\right) \in \mathcal{X} \times \mathcal{X}} \sum_{p=1}^{\infty} \frac{1}{p} \frac{1-q_{1}^{p}}{1-q_{2}^{-p}}\left(c_{\mathrm{i}\left(x_{L}\right), \mathrm{i}\left(x_{R}\right)}^{+}\right)^{[p]} x_{L}^{-p} x_{R}^{p}\right) \\
& \times \exp \left(\sum_{x \in \mathcal{X}}\left(-\frac{\kappa_{\mathrm{i}(x)}}{2}\left(\log _{q_{2}} x-1\right) \log _{q_{2}} x+\log \mathfrak{q}_{\mathrm{i}(x)} \log _{q_{2}} \frac{x}{\dot{x}}+\sum_{p=1}^{\infty}\left(1-q_{1}^{p}\right) t_{\mathrm{i}(x), p} x^{p}\right)\right)
\end{aligned}
$$

where $\stackrel{\circ}{x}_{i, \alpha, s_{1}}=\nu_{i, \alpha} q_{1}^{s_{1}-1}$ denotes ground configuration of the empty partition $\lambda=0$, so that the $\log _{q_{2}} \frac{x}{\grave{x}}$ counts the size of the partition $\lambda$ equal to the instanton charge $\mathrm{k}$. In particular, the vector and bifundamental hypermultiplet contributions are generated explicitly by the first factor in (3.21) and are given by

$$
\begin{aligned}
Z_{i}^{\mathrm{vec}} & =\prod_{\left(x, x^{\prime}\right) \in \mathcal{X}_{i} \times \mathcal{X}_{i}}\left(q \frac{x}{x^{\prime}} ; q_{2}\right)_{\infty}\left(q_{2} \frac{x}{x^{\prime}} ; q_{2}\right)_{\infty}^{-1}, \\
Z_{e: i \rightarrow j}^{\mathrm{bf}} & =\prod_{\left(x, x^{\prime}\right) \in \mathcal{X}_{i} \times \mathcal{X}_{j}}\left(\mu_{e}^{-1} q \frac{x}{x^{\prime}} ; q_{2}\right)_{\infty}^{-1}\left(\mu_{e}^{-1} q_{2} \frac{x}{x^{\prime}} ; q_{2}\right)_{\infty},
\end{aligned}
$$

These factors correspond to the localization determinants in the full Nekrasov partition function in quiver gauge theory, including the perturbative one-loop factor (see for example [2]). The $\kappa$-term in the second factor in (3.21) accounts for the Chern-Simons contribution, the $\mathfrak{q}$-term in the second factor in (3.21) accounts for the instanton number counting parameter, and the $t$-terms in the second factor in (3.21) account for the deformation of the Nekrasov partition function by the higher times.

Remark. In the limit $q_{2} \rightarrow 1$ the extended partition function is dominated by the critical set $\mathcal{X}_{\text {crit }}$ determined in [2] and the variables $x \in \mathcal{X}_{\text {crit }}$ satisfy the Bethe equations. 
3.12. Reflection of the index. Let $\mathbf{X}^{[p]}$ be $p$-th Adams operation applied to an object $\mathbf{X}$. In terms of the decomposition of $\mathbf{X}$ into the Chern roots $\mathbf{X}=\sum x$, the $p$-th Adams operation takes each Chern root $x$ into the $p$-th power, so that $\mathbf{X}^{[p]}=\sum x^{p}$. Then the following reflection equation holds

$$
\exp \left(\sum_{p=1}^{\infty} \frac{1}{p}\left[\left(\mathbf{X}^{\vee}\right)^{[p]}\right]\right)=(-1)^{\mathrm{rk} \mathbf{X}}[\operatorname{det} \mathbf{X}] \exp \left(\sum_{p=1}^{\infty} \frac{1}{p}\left[\mathbf{X}^{[p]}\right]\right)
$$

3.13. The ordered partition function. Pick an order $\succ$ on the set $\mathcal{X}$. For example, an order can be chosen by taking $\left|q_{1}\right| \ll\left|q_{2}^{-1}\right|<1$ and $|\nu| \simeq|\mu| \simeq 1$. Then define $x_{L} \succ x_{R}$ if $\left|x_{L}\right|>\left|x_{R}\right|$, which is basically the radial order in CFT. The sum over all pairs $\left(x_{L}, x_{R}\right) \in$ $\mathcal{X} \times \mathcal{X}$ in the partition function (3.21) can be transformed to the sum over pairs $\left(x_{L} \succ x_{R}\right)$, over pairs $\left(x_{L} \prec x_{R}\right)$ and the diagonal pairs $\left(x_{L}=x_{R}\right)$. The diagonal part gives $(\mathfrak{q}, \nu, \mu, t)$ independent factor that we omit. The sum over pairs $\left(x_{L} \succ x_{R}\right)$ and $\left(x_{L} \prec x_{R}\right)$ can be combined together using the reflection equation (3.24)

$$
\begin{aligned}
& Z_{\mathrm{T}}(t)=\sum_{\mathcal{X} \in \mathfrak{M}^{\top}} \exp \left(\sum_{\left(x_{L} \succ x_{R}\right) \in \Lambda^{2} \mathcal{X}}-\left(c_{\mathrm{i}\left(x_{R}\right), \mathrm{i}\left(x_{L}\right)}\right)^{[0]} \beta \log \frac{x_{R}}{x_{L}}-\sum_{p=1}^{\infty} \frac{1}{p} \frac{1-q_{1}^{p}}{1-q_{2}^{-p}}\left(c_{\mathrm{i}\left(x_{L}\right), \mathrm{i}\left(x_{R}\right)}\right)^{[p]} \frac{x_{R}^{p}}{x_{L}^{p}}\right) \\
& \times \exp \left(\sum_{x \in \mathcal{X}}\left(-\frac{\kappa_{\mathrm{i}(x)}}{2}\left(\log _{q_{2}} x-1\right) \log _{q_{2}} x+\log \mathfrak{q}_{\mathrm{i}(x)} \log _{q_{2}} \frac{x}{\dot{x}}+\sum_{p=1}^{\infty}\left(1-q_{1}^{p}\right) t_{\mathrm{i}(x), p} x^{p}\right)\right)
\end{aligned}
$$

where $\beta=-\frac{\log q_{1}}{\log q_{2}}=-\frac{\epsilon_{1}}{\epsilon_{2}}$, and the mass deformed Cartan matrix is defined

$$
c_{i j}=c_{i j}^{+}+c_{i j}^{-}, \quad c_{i j}^{-}=q^{-1}\left(c_{j i}^{+}\right)^{\vee}, \quad c_{i j}=\left(1+q^{-1}\right) \delta_{i j}-\sum_{e: i \rightarrow j} \mu_{e}^{-1}-\sum_{e: j \rightarrow i} \mu_{e} q^{-1}
$$

which is symmetric up to conjugation and the $q$-factor,

$$
c_{j i}=q^{-1} c_{i j}^{\vee}
$$

3.14. Extended partition function is a state. The exponentiated sum over the pairs $\left(x_{L} \succ x_{R}\right)$ in the equation (3.25) suggests a natural way to present the extended partition function $Z_{\mathrm{T}}(t)$ as a state $\left|Z_{\mathrm{T}}\right\rangle$ in the infinite-dimensional T-character valued Fock space ch $\operatorname{Rep}_{\mathrm{T}}[[t]]$. The Fock space ch $\operatorname{Rep}_{\mathrm{T}}[[t]]$ is Verma module for the Heisenberg algebra $\mathbf{H}$ generated by the operators $\left(\partial_{i, p}\right)_{i \in \Gamma_{0}, p \in[0 \ldots \infty]}$ and $t=\left(t_{i, p}\right)_{i \in \Gamma_{0}, p \in[0 \ldots \infty]}$ over ch Rep $\operatorname{T}_{\mathrm{T}}$ with canonical commutators

$$
\left[\partial_{i, m}, t_{j, n}\right]=\delta_{i j} \delta_{m n}
$$

where

$$
t_{i, 0}=\log _{q_{2}} \mathfrak{q}_{i}
$$

The elements of the Fock space ch $\operatorname{Rep}_{\mathrm{T}}[[t]]$ are formal $t$-series valued in the ring of $\mathrm{T}$ characters. The $t$-constants are lowest-weight states (vacua); they are annihilated by all lowering operators $\partial_{i, p}$. A state in the Fock space ch Rep $[$ T $[t]]$ can be obtained by an action of an operator in the algebra $\mathbf{H}$ on the vacuum $|1\rangle$. 
3.15. Free bosons and vertex operators. The state $\left|Z_{\mathrm{T}}\right\rangle$ can be presented as

$$
\left|Z_{\mathrm{T}}\right\rangle=\sum_{\mathcal{X} \in \mathfrak{M}^{\top}} \prod_{x \in \mathcal{X}}^{\succ} S_{\mathrm{i}(x), x}|1\rangle
$$

where $\prod^{\succ}$ denotes the $\succ$-ordered product over $x \in \mathcal{X}$ of the vertex operators

$$
S_{i, x}=: \exp \left(\sum_{p>0} s_{i,-p} x^{p}+s_{i, 0} \log x+\tilde{s}_{i, 0}+\sum_{p>0} s_{i, p} x^{-p}\right):
$$

Here the free field modes or oscillators are

$$
s_{i,-p} \stackrel{p>0}{=}\left(1-q_{1}^{p}\right) t_{i, p}, \quad s_{i, 0}=t_{i, 0}, \quad s_{i, p} \stackrel{p>0}{=}-\frac{1}{p} \frac{1}{1-q_{2}^{-p}} c_{j i}^{[p]} \partial_{j, p}
$$

with commutation relations

$$
\left[s_{i, p}, s_{j, p^{\prime}}\right]=-\delta_{p+p^{\prime}, 0} \frac{1}{p} \frac{1-q_{1}^{p}}{1-q_{2}^{-p}} c_{j i}^{[p]} \quad(p>0)
$$

The conjugate zero mode $\tilde{s}_{i, 0}$ satisfies

$$
\left[\tilde{s}_{i, 0}, s_{j, p}\right]=-\beta \delta_{0, p} c_{j i}^{[0]}
$$

The normal product notation : $e^{A_{1}} e^{A_{2}}$ :, where operators $A_{1}, A_{2}$ are linear in the free fields, means that all operators $\left(s_{i, p}\right)_{p \leq 0}$ are placed to the left of $\left(s_{i, p}\right)_{p>0}$ and $\tilde{s}_{i, p}$.

The relations (3.34) and (3.33), and the relation $e^{A_{1}} e^{A_{2}}=e^{\left[A_{1}, A_{2}\right]} e^{A_{2}} e^{A_{1}}$ for central $\left[A_{1}, A_{2}\right]$, imply that the operator-state representation of the partition function (3.30) is equivalent to the quiver gauge theory definition (3.25) if gauge theory couplings $\kappa_{i}$ and $\mathfrak{q}_{i}$ are evaluated as

$$
\kappa_{i}=-\mathrm{n}_{j}\left(c_{j i}^{-}\right)^{[0]}, \quad \log _{q_{2}} \mathfrak{q}_{i}=\beta+t_{i, 0}+\mathrm{n}_{j}\left(c_{j i}^{-}\right)^{\left[\log _{q_{2}}\right]}-\log _{q_{2}}\left((-1)^{\mathrm{n}_{j}} \nu_{j}\right)\left(c_{j i}^{-}\right)^{[0]}
$$

where

$$
\left(c_{i j}^{-}\right)^{\left[\log _{q_{2}}\right]}=\delta_{i j} \log _{q_{2}} q^{-1}-\sum_{e: j \rightarrow i} \log _{q_{2}}\left(\mu_{e} q^{-1}\right)
$$

3.16. Screening charges. The configuration sets $\mathcal{X} \in \mathfrak{M}^{\top}$ are described by the partitions, which are explicitly collections of constrained sequences

$$
\left(\lambda_{i, \alpha, 1} \geq \lambda_{i, \alpha, 2} \geq \cdots \geq 0=0=0=\ldots\right)_{i \in \Gamma_{0}, \alpha \in\left[1 \ldots \mathrm{n}_{i}\right]}
$$

Let $\mathcal{X}_{0, i}$ be the ground configuration with all $\lambda_{i, \alpha, *}=0$, and define its union $\mathcal{X}_{0}=\bigsqcup_{i \in \Gamma_{0}} \mathcal{X}_{0, i}$. Let $\mathbb{Z}^{\mathcal{X}_{0, i}}$ be the set of collections of arbitrary integer sequences terminating by zeroes

$$
\left(\lambda_{i, \alpha, s_{1}} ? \lambda_{i, \alpha, s_{2}} ? \ldots ?=0=0=\ldots\right)_{i \in \Gamma_{0}, \alpha \in\left[1 \ldots \mathrm{n}_{i}\right]}
$$

Then $\mathfrak{M}^{\top} \subset \mathbb{Z}^{\mathcal{X}_{0}}$. It turns out that the summation over $\mathfrak{M}^{\top}$ in (3.30) can be extended to the whole $\mathbb{Z}_{\geq 0}^{\mathcal{X}_{0}}$ without changing the result because

$$
\prod_{x \in \mathcal{X}} S_{\mathrm{i}(x), x}|1\rangle=0 \quad \text { if } \mathcal{X} \in \mathbb{Z}^{\mathcal{X}_{0}} \text { but } \mathcal{X} \notin \mathfrak{M}^{\top}
$$


due to the zero factors in the normal ordering product of vertex operators $S_{i, x}$ for the sequences $\left(x_{i, \alpha, s_{1}}=\nu_{i, \alpha} q_{1}^{s_{1}-1} q_{2}^{\lambda_{i, \alpha, s_{1}}}\right)$ where $\lambda$ does not satisfy the constraint (3.37). Therefore

$$
\left|Z_{\mathrm{T}}\right\rangle=\sum_{\mathcal{X} \in \mathbb{Z}^{\mathcal{X}_{0}}} \prod_{x \in \mathcal{X}}^{\succ} S_{\mathrm{i}(x), x}|1\rangle
$$

For every point $\dot{x}_{i, \alpha, s_{1}}=\nu_{i, \alpha} q_{1}^{s_{1}-1}$ in the ground configuration $\mathcal{X}_{0}$ defines the operator called screening charge

$$
\mathrm{S}_{i, \grave{x}}=\sum_{s_{2} \in \mathbb{Z}} S_{i, q_{2}^{s_{2}} \dot{x}}
$$

Then the state $\left|Z_{\mathrm{T}}\right\rangle$ is obtained by applying to the vacuum the ordered product of $\mathrm{S}_{i}$ operators

$$
\left|Z_{\mathrm{T}}\right\rangle=\prod_{\dot{x} \in \mathcal{X}_{0}}^{\succ} \mathrm{S}_{\mathrm{i}(\tilde{x}), \dot{x}}|1\rangle
$$

The partition function of plain, not $t$-extended theory, can be interpreted as the projection

$$
\left\langle 1 \mid Z_{\mathrm{T}}\right\rangle=\left\langle 1\left|\prod_{\dot{x} \in \mathcal{X}_{0}}^{\succ} \mathrm{S}_{\mathrm{i}(\mathfrak{x}), \dot{x}}\right| 1\right\rangle
$$

since the dual vacuum obeys $\langle 1| t_{i, n}=0$ for $n \geq 1, \forall i \in \Gamma_{0}$.

3.17. The Ward identities. The sum representation of the operator $S_{i, x}$ in (3.41) is explicitly invariant under the $\mathbb{Z}$-translational symmetry $s_{2} \rightarrow s_{2}+\mathbb{Z}$ (change of variables). Hence the representation of the partition function (3.42) is invariant under the $\mathbb{Z}^{\mathcal{X}_{0}}$ symmetry that shifts the summation variables $s_{2}$ for each $\dot{x}_{i, \alpha, s_{1}}$. In the $\mathcal{S}$ space-time picture the variation $s_{2} \rightarrow s_{2}+1$ amounts to the $z_{2}$ multiplicative change of variables in the $z_{1}, z_{2}$-mode expansion $\phi_{i, \alpha, s_{1}} z_{1}^{s_{1}} \rightarrow z_{2} \phi_{i, \alpha, s_{1}} z_{1}^{s_{1}}$ where $\phi$ is in the sheaf $\mathcal{Y}$. The shift $s_{2} \rightarrow s_{2}+1$ adds one box to the partition, or equivalently one instanton to the gauge field on the space-time.

3.18. The $\mathbf{Y}$-operators. In [2, 7] the $\left(\mathbf{Y}_{i, x}\right)_{i \in \Gamma_{0}}$ observables were introduced in the K-theory of the moduli space $\mathfrak{M}^{\top}$ of the quiver gauge theory

$$
\mathbf{Y}_{i, x}:=\exp \left(-\sum_{p=1}^{\infty} \frac{x^{-p}}{p} \mathbf{Y}_{i}^{[p]}\right)
$$

The expectation value of the observable $\mathbf{Y}_{i, x}$ in the plain (not $t$-extended) theory is computed by the pushforward integration over the moduli space $\mathfrak{M}^{\top}(2.10)$

$$
\left\langle\mathbf{Y}_{i, x}\right\rangle:=\operatorname{ch}_{\mathrm{T}} \pi_{!} \mathfrak{q}^{\gamma} \mathbf{Y}_{i, x}
$$

It is natural to lift the $\mathbf{Y}_{i, x}$ observables to the $t$-extended theory by giving them the operator definition:

$$
\mathbf{Y}_{i, x}=q_{1}^{\tilde{\rho}_{i}}: \exp \left(\sum_{p>0} y_{i,-p} x^{p}+y_{i, 0}+\sum_{p>0} y_{i, p} x^{-p}\right):
$$


where $\tilde{\rho}_{i}:=\sum_{j \in \Gamma_{0}} \tilde{c}_{j i}^{[0]}$ are components of the Weyl vector in the basis of simple roots. If the quiver is the affine type, we put $\tilde{\rho}_{i}=0$. The operator $Y_{i, x}$ is an element of the Heisenberg algebra $\mathbf{H}$. The oscillators $y_{i, p}$ are expressed in terms of $t_{i, p}$ and $\partial_{i, p}$

$$
(p>0) \quad y_{i,-p}=\left(1-q_{1}^{p}\right)\left(1-q_{2}^{p}\right) \tilde{c}_{j i}^{[-p]} t_{j, p}, \quad y_{i, 0}=-t_{j, 0} \tilde{c}_{j i}^{[0]} \log q_{2} \quad y_{i, p}=-\frac{1}{p} \partial_{i, p}
$$

or equivalently terms of the free field $s_{i, p}$

$$
y_{i, p} \stackrel{p \neq 0}{=}\left(1-q_{2}^{-p}\right) s_{j, p} \tilde{c}_{j i}^{[p]}, \quad y_{i, 0}=\left(\log q_{2}^{-1}\right) s_{j, 0} \tilde{c}_{j i}^{[0]}
$$

where $\tilde{c}_{i j}$ is the inverse to the mass-deformed Cartan matrix $c_{i j}$ defined in (3.26). The definitions (3.47) and (2.10) imply

$$
\left\langle\mathbf{Y}_{i, x}\right\rangle=\left\langle 1\left|\mathbf{Y}_{i, x}\right| Z_{\mathrm{T}}\right\rangle
$$

3.19. The OPE of $Y$ and $S$. The commutation relations between $y_{i, p}$ and $s_{j, p^{\prime}}$ are

$$
\left[y_{i, p}, s_{j, p^{\prime}}\right]=-\frac{1}{p}\left(1-q_{1}^{p}\right) \delta_{p+p^{\prime}, 0} \delta_{i j}, \quad\left[\tilde{s}_{i, 0}, y_{j, 0}\right]=-\delta_{i j} \log q_{1}
$$

Then (3.49) can be also seen from the commutation relations (3.50) and normal ordering because at $|x|>\left|x^{\prime}\right|$ we have

$$
\mathrm{Y}_{i, x} S_{i, x^{\prime}}=\frac{1-x^{\prime} / x}{1-q_{1} x^{\prime} / x}: \mathrm{Y}_{i, x} S_{i, x^{\prime}}: \quad \mathrm{Y}_{i, x} S_{j, x^{\prime}}=: \mathrm{Y}_{i, x} S_{j, x^{\prime}}: \quad i \neq j
$$

Therefore at each fixed point configuration $\mathcal{X} \in \mathfrak{M}^{\top}$

$$
\left\langle 1\left|\mathrm{Y}_{i, x} \prod_{x^{\prime} \in \mathcal{X}}^{\succ} S_{\mathrm{i}(x), x^{\prime}}\right| 1\right\rangle=q_{1}^{\tilde{\rho}_{i}}\left(\prod_{x^{\prime} \in \mathcal{X}_{i}} \frac{1-x^{\prime} / x}{1-q_{1} x^{\prime} / x}\right)\left\langle 1\left|\prod_{x^{\prime} \in \mathcal{X}}^{\succ} S_{\mathrm{i}(x), x^{\prime}}\right| 1\right\rangle
$$

like in the definition that was given in [2]. The observable $Y_{i, x}$ is not regular in the $\mathbb{C}_{x}^{\times}$ because of the possible poles at points $x=q_{1} x^{\prime}$.

3.20. The commutator of $Y$ and $S$. The commutation relations (3.50) also imply for $\left|x^{\prime}\right|>|x|$

$$
S_{i, x^{\prime}} \mathrm{Y}_{i, x}=q_{1}^{-1} \frac{1-x / x^{\prime}}{1-q_{1}^{-1} x / x^{\prime}}: S_{i, x^{\prime}} \mathrm{Y}_{i, x}:
$$

Therefore (3.51) and (3.53) imply the non-zero radial-ordered commutator

$$
\left[\mathrm{Y}_{i, x}, S_{i, x^{\prime}}\right]=\left(1-q_{1}^{-1}\right) \delta\left(q_{1} \frac{x^{\prime}}{x}\right): S_{i, x^{\prime}} \mathrm{Y}_{i, x}:
$$

where by definition $\delta(z)=\sum_{n \in \mathbb{Z}} z^{n}$.

The fact that observable $\mathrm{Y}_{i, x}$ has singularities at $x=q_{1} x^{\prime}$ in (3.53) is equivalent to the presence of the $\delta\left(q_{1} x^{\prime} / x\right)$ in the radial ordered commutator between $\mathrm{Y}_{i, x}$ and $S_{i, x^{\prime}}$. This is a general statement implied by Cauchy integral formula and familiar from the formalism of radial quantization in CFT. 
3.21. The V-operators. We introduce another kind of vertex operator to reproduce the fundamental matter contribution in gauge theory. As explained before, this contribution is given by shift of the time variables (3.9), which can be implemented by the operator

$$
\mathrm{V}_{i, x}=: \exp \left(\sum_{p>0} v_{i,-p} x^{p}+\sum_{p>0} v_{i, p} x^{-p}\right):
$$

The corresponding free field is explicitly written

$$
(p>0) \quad v_{i,-p}=-t_{j, p} \tilde{c}_{j i}^{[-p]} \quad v_{i, p}=\frac{1}{p} \frac{1}{\left(1-q_{1}^{p}\right)\left(1-q_{2}^{p}\right)} \partial_{i, p} .
$$

We remark a simple relation to the $y$-operators (3.47)

$$
v_{i, p}=-\frac{1}{\left(1-q_{1}^{p}\right)\left(1-q_{2}^{p}\right)} y_{i, p} .
$$

Then the OPE of $\mathrm{V}$ and $S$ operators are given by

$$
\mathrm{V}_{i, x} S_{i, x^{\prime}}=\left(\frac{x^{\prime}}{x} ; q_{2}\right)_{\infty}^{-1}: \mathrm{V}_{i, x} S_{i, x^{\prime}}:, \quad S_{i, x^{\prime}} \mathrm{V}_{i, x}=\left(\frac{q_{2} x}{x^{\prime}} ; q_{2}\right)_{\infty}: \mathrm{V}_{i, x} S_{i, x^{\prime}}:
$$

corresponding to the fundamental and antifundamental hypermultiplet contributions. Thus the extended partition function in the presence of (anti)fundamental matters is obtained by inserting the $\mathrm{V}$-operators

$$
\left|Z_{\mathrm{T}}\right\rangle=\left(\prod_{x \in \mathcal{X}_{\mathrm{f}}} \mathrm{V}_{\mathrm{i}(x), x}\right)\left(\prod_{\tilde{x} \in \mathcal{X}_{0}}^{\succ} \mathrm{S}_{\mathrm{i}(\tilde{x}), \check{x}}\right)\left(\prod_{x \in \tilde{\mathcal{X}}_{\mathrm{f}}} \mathrm{V}_{\mathrm{i}(x), x}\right)|1\rangle
$$

where $\mathcal{X}_{\mathrm{f}}=\left\{\mu_{i, f}\right\}_{i \in \Gamma_{0}, f \in\left[1 \ldots \mathrm{n}_{i}^{\mathrm{f}}\right]}$ and $\tilde{\mathcal{X}}_{\mathrm{f}}=\left\{\tilde{\mu}_{i, f}\right\}_{i \in \Gamma_{0}, f \in\left[1 \ldots \tilde{n}_{i}^{\mathrm{f}}\right]}$ are sets of fundamental and antifundamental mass parameters. This $\mathrm{V}$-operator creates a singularity on the curve at $x=\mu_{i, f}$. Then the plain partition function $(t=0)$ is given as a correlator as shown in (3.43),

$$
Z_{\mathrm{T}}(t=0)=\left\langle 1 \mid Z_{\mathrm{T}}\right\rangle=\left\langle 1\left|\left(\prod_{x \in \mathcal{X}_{\mathrm{f}}} \mathrm{V}_{\mathrm{i}(x), x}\right)\left(\prod_{\dot{x} \in \mathcal{X}_{0}}^{\succ} \mathrm{S}_{\mathrm{i}(\dot{x}), x}\right)\left(\prod_{x \in \tilde{\mathcal{X}}_{\mathrm{f}}} \mathrm{V}_{\mathrm{i}(x), x}\right)\right| 1\right\rangle .
$$

\section{W-ALGEBRA}

Here we describe the construction of regular observables $T$ of the extended gauge theory and explain isomoprhism with Shiraishi et al. [5] and Frenkel-Reshetikhin [1] definition of $W_{q_{1}, q_{2}}$ algebra as commutant of screening charges in the Heisenberg algebra $\mathbf{H}$, and define K-theoretical quiver W-algebra for $\mathcal{S}=\mathbb{C}_{q_{1}, q_{2}}$.

4.1. Pole cancellation in $T: A_{1}$-example. Consider the simplest quiver $\Gamma=A_{1}$ for example. In [2] in the study of the $q_{2}=1$ limit of the gauge theory partition function, motivated by cut-crossing story of [7], it was suggested to consider the observable 3

$$
T_{1, x}=\mathrm{Y}_{1, x}+\mathrm{Y}_{1, q^{-1} x}
$$

\footnotetext{
${ }^{3}$ We adopted the normalizations and the zero modes to the conventions of the present paper in which the $T$-observables have the simplest canonical form.
} 
for its virtue of being regular function in $\mathbb{C}_{x}^{\times}$. This is the simplest example of $q$-character representing the $T$-matrix of Baxter coming from $\mathbf{U}_{q}\left(\hat{\mathfrak{s l}}_{2}\right)$-integrable system and Baxter equation.

In fact, the same observable $T_{1, x}$ remains regular function of $x$ for generic $q_{2}$. Indeed, in the operator formalism we find

$$
\begin{aligned}
\mathrm{Y}_{1, x} S_{1, x^{\prime}} & =\frac{1-x^{\prime} / x}{1-q_{1} x^{\prime} / x}: \mathrm{Y}_{1, x} S_{1, x^{\prime}}: \\
\mathrm{Y}_{1, q^{-1} x}^{-1} S_{1, x^{\prime \prime}} & =\frac{1-q q_{1} x^{\prime \prime} / x}{1-q x^{\prime \prime} / x}: \mathrm{Y}_{1, q^{-1} x}^{-1} S_{1, x^{\prime \prime}}:
\end{aligned}
$$

so the potential singularity in the first line is for $x^{\prime}=q_{1}^{-1} x$ and in the second line for $x^{\prime \prime}=q^{-1} x$. Therefore, the two singularities have chance to cancel at $x^{\prime}=q_{2} x^{\prime \prime}$. Recall that the state $\left|Z_{\mathrm{T}}\right\rangle$ is obtained with the sums (3.41) and there is internal symmetry for the shift of the summation indexing variable (see Ward identity in Sec. 3.17) so that for every term $S_{1, x^{\prime \prime}}$ there is a term with $S_{1, x^{\prime}}$ with $x^{\prime}=q_{2} x^{\prime \prime}$.

Indeed, we find for the first term the normal ordered expression

$$
: Y_{1, x} S_{1, q_{1}^{-1} x}:=: \exp \left(-\frac{1}{2} s_{1,0} \log q_{2}+s_{1,0} \log \left(x q_{1}^{-1}\right)+\tilde{s}_{1,0}+\sum_{p \neq 0}\left(q_{1}^{p}+\frac{1-q_{2}^{-p}}{1+q^{-p}}\right) s_{1, p} x^{-p}\right):
$$

and for the second term the normal ordered expression

$q_{1}: \mathrm{Y}_{1, q^{-1} x}^{-1} S_{1, q^{-1} x}:=: \exp \left(+\frac{1}{2} s_{1,0} \log q_{2}+s_{1,0} \log \left(x q^{-1}\right)+\tilde{s}_{1,0}+\sum_{p \neq 0}\left(q^{p}-\frac{1-q_{2}^{-p}}{1+q^{-p}} q^{p}\right) s_{1, p} x^{-p}\right):$

which are exactly identical. The respective residues in the prefactors (4.2) are $\left(1-q_{1}^{-1}\right)$ and $\left(q_{1}^{-1}-1\right)$ which respectively cancel each other. This computation proves regularity in $x \in \mathbb{C}^{\times}$ of the state $\left|Z_{\mathrm{T}}\right\rangle$ of higher $t$-extended gauge theory in $A_{1}$ example

$$
\partial_{\bar{x}} T_{1, x}\left|Z_{\mathrm{T}}\right\rangle=0
$$

4.2. Commutator of $T$ and $\mathrm{S}$ vanishing: $A_{1}$-example. An exactly equivalent presentation of the regularity of $T_{1, x}$ is the statement that

$$
\left[T_{1, x}, \mathrm{~S}_{1, x^{\prime}}\right]=0
$$

where $S_{1, x^{\prime}}$ is the screening charge (3.41) defined as the summation over the $q_{2}^{\mathbb{Z}}$ shifts. Indeed, we have

$$
\begin{gathered}
{\left[\mathrm{Y}_{1, x}, S_{1, x^{\prime}}\right]=\left(1-q_{1}^{-1}\right) \delta\left(q_{1} \frac{x^{\prime}}{x}\right): \mathrm{Y}_{1, x} S_{1, x^{\prime}}:} \\
{\left[\mathrm{Y}_{1, q^{-1} x}^{-1}, S_{1, x^{\prime}}\right]=\left(q_{1}^{-1}-1\right) \delta\left(q \frac{x^{\prime}}{x}\right): \mathrm{Y}_{1, q^{-1} x} S_{1, x^{\prime}}:}
\end{gathered}
$$

The total sum is $q_{2}$-difference which cancels after summation over $q_{2}^{\mathbb{Z}}$ shifts entering definition of screening charge (3.41). This is the consequence of the Ward identity in Sec. 3.17. 
4.3. W-algebra of $A_{1}$-quiver. Consequently the operator $T_{1, x}$ can be moved in the position in the radial-ordered operator-state presentation of the extended gauge theory partition state (3.42)

$$
T_{1, x}|1\rangle=T_{1, x} \mathrm{~S}_{1, x^{\prime}} \mathrm{S}_{1, x^{\prime \prime}} \ldots|1\rangle=\mathrm{S}_{1, x^{\prime}} T_{1, x} \mathrm{~S}_{1, x^{\prime \prime}} \ldots|1\rangle=\mathrm{S}_{1, x^{\prime}} \mathrm{S}_{1, x^{\prime \prime}} T_{1, x} \ldots|1\rangle
$$

The operators $\mathrm{S}_{i, x^{\prime}}$ can be thought as exponentiated Hamiltonians of the $q_{1}, q_{2}$-deformed CFT. The commutant of the Hamiltonians is the conserved current $T_{1, x}$ which is regular

$$
\partial_{\bar{x}} T_{1, x}=0
$$

Consequently, $T_{1, x}$ has well defined, time-radial independent, modes

$$
T_{1, x}=\sum_{p \in \mathbb{Z}} T_{1,[p]} x^{-p}
$$

We define the algebra $W_{q_{1}, q_{2}}\left(A_{1}\right)$, a.k.a. the $q$-deformed Virasoro algebra, to be the subalgebra in $\mathbf{H}$ generated by the modes of the conserved current $T_{1, x}$. This definition is in the exact agreement with Shiraishi et al. [5] and Frenkel-Reshetikhin [1].

4.4. W-algebra of quiver: definition. The definition of the state $\left|Z_{\mathrm{T}}\right\rangle(\underline{3.42})$ implies that the current $T_{i, x}$ is regular

$$
\partial_{\bar{x}} T_{i, x}\left|Z_{\mathrm{T}}\right\rangle=0
$$

if it commutes with all screening operators:

$$
\left[T_{i, x}, \mathrm{~S}_{j, x^{\prime}}\right]=0 \quad j \in \Gamma_{0}, \quad x^{\prime} \in \mathcal{X}_{j}
$$

This explains isomorphism between the gauge theoretic construction of $q_{1} q_{2}$-characters [2, 3] and definition of $W_{q_{1}, q_{2}}$-algebras [1, 5, 9] as the algebra generated by currents $\left(T_{i, x}\right)_{i \in \Gamma_{0}}$ which are defined as commutants of screening charges $\left(S_{i}\right)_{i \in \Gamma_{0}}$ in the vertex operator algebra defined by the free fields from Heisenberg algebra $\mathbf{H}$ and expressed as

$$
T_{i, x}=Y_{i, x}+\ldots
$$

We define in the same way the $\mathrm{W}$-algebra $W(\Gamma, \mathcal{S})$ for generic quiver $\Gamma$ with generalized even symmetric Borcherds-Kac-Moody-Cartan matrix, mass deformed by $\mu: \Gamma_{1} \rightarrow \mathbb{C}^{\times}$, as in equation (3.26), and for $\mathcal{S}=\mathbb{C}_{q_{1}, q_{2}}$ as the algebra generated by currents $T_{i, x}$ commuting with all screening charges $\left(\mathrm{S}_{i, x}\right)_{i \in \Gamma_{0}}$, or equivalently, regular on the higher times extended gauge theory state (4.11). We expect to generalize the definition for more general and possibly higher dimensional varieties $\mathcal{S}$.

\section{EXAMPLES}

We consider a few examples to illustrate the equivalence between gauge-theory formalism [2, 3, 7] and the operator formalism [1, 5, 9, 15, 16]. 
5.1. Commutator of $T$ and $S$ vanishing: general quiver, local reflection. Suppose that there is no edge loop from a node $i$ to itself and consider

$$
T_{i, x}=\mathrm{Y}_{i, x}+: \mathrm{Y}_{i, q^{-1} x}^{-1} \prod_{e: i \rightarrow j} \mathrm{Y}_{j, \mu_{e}^{-1} x} \prod_{e: j \rightarrow i} \mathrm{Y}_{j, q^{-1} \mu_{e} x}:+\ldots
$$

The vanishing of commutator

$$
\left[T_{i, x}, \mathrm{~S}_{i, x^{\prime}}\right]=0
$$

follows from (3.54) and the relation

$$
q_{1}: \mathrm{Y}_{i, q^{-1} x}^{-1}\left(\prod_{e: i \rightarrow j} \mathrm{Y}_{j, \mu_{e}^{-1} x} \prod_{e: j \rightarrow i} \mathrm{Y}_{j, q^{-1} \mu_{e} x}\right) S_{i, q^{-1} x}:=: \mathrm{Y}_{i, x} S_{i, q_{1}^{-1} x}:
$$

Indeed, this relation is equivalent to

$$
q_{1}^{-1}: Y_{i, x} \mathrm{Y}_{i, q^{-1} x}\left(\prod_{e: i \rightarrow j} \mathrm{Y}_{j, \mu_{e}^{-1} x} \prod_{e: j \rightarrow i} \mathrm{Y}_{j, q^{-1} \mu_{e} x}\right)^{-1}:=: S_{i, q^{-1} x} S_{i, q_{1}^{-1} x}^{-1}:
$$

which simply expresses the defining relation (3.48) between $\mathrm{Y}_{i, x}$ and $S_{j, x}$ in the exponentiated form: the field $y_{i}(x)$ (of the Cartan weight type) is the $q_{2}$-derivative of the field $s_{i}(x)$ (of the Cartan root type). Namely, the relation (5.4) is the identity

$$
\log q_{2}^{-1} s_{k, 0} \tilde{c}_{k j}^{[0]} c_{j i}^{[0]}+\sum_{p \neq 0}\left(1-q_{2}^{-p}\right) q^{p} s_{k, p} \tilde{c}_{k j}^{[p]} c_{j i}^{[p]} x^{-p}=\left(\log \left(x q^{-1}\right)-\log \left(x q_{1}^{-1}\right)\right) s_{i, 0}+\sum_{p \neq 0}\left(q^{p}-q_{1}^{p}\right) s_{i, p} x^{-p}
$$

thanks to the definition of the $\mu$-dependent Cartan matrix $c_{i j}(3.26)$ and its inverse $\tilde{c}_{i j}$ so that $\tilde{c}_{k j}^{[p]} c_{j i}^{[p]}=\delta_{k i}$. This leads to

$$
\left[: \mathrm{Y}_{i, q^{-1} x}^{-1} \prod_{e: i \rightarrow j} \mathrm{Y}_{j, \mu_{e}^{-1} x} \prod_{e: j \rightarrow i} \mathrm{Y}_{j, q^{-1} \mu_{e} x}:, S_{i, x^{\prime}}\right]=\left(q_{1}^{-1}-1\right) \delta\left(q \frac{x^{\prime}}{x}\right): \mathrm{Y}_{1, q^{-1} x} S_{1, x^{\prime}}:
$$

and therefore (5.2) holds at the level of the first two terms. The second term contains the $\mathrm{Y}_{j}$ fields for the nodes $j$ linked to the node $i$. This term might give potential singularities, or, equivalently, $\delta$-functions in the commutators associated to the $S_{i}$ operators. Then one needs to continue to apply Weyl reflections to generate terms which cancel the singularities. The algebraic structure is associated to highest weight Verma module of the generalized Borcherds-Kac-Moody algebra $\mathfrak{g}_{\Gamma}$. If $\Gamma$ is of finite Dynkin type the process terminates, the associated Verma module is finite-dimensional. The finite-dimensional case was studied in details in [9].

More generally, for infinite-dimensional Verma module, the recursive algorithm is also applicable which builds a tree starting from the root node $Y_{i, x}$. The vertices of the tree are monomials in the $T_{i, x}$ current, and the edges are colored by the nodes $i$ of the quiver. Two monomials are linked by edge of color $i$ if they are related by the local reflection move (5.1). The algorithm can be computerized.

Alternatively, Nekrasov presented closed formula [3] which express the $q_{1} q_{2}$-characters in terms of geometry of Nakajima's quiver variety [17, 42]. This formula can be thought as $q_{2}$-deformation of original Nakajima's construction of $q$-characters of $\mathbf{U}_{q}\left(L \mathfrak{g}_{\Gamma}\right)$ from the $q$ equivariant K-theory on the quiver variety $\mathfrak{M}_{\mathbf{w}, \mathbf{v}}^{\mathrm{Nak}}=T_{q}^{*} \mathfrak{M}_{\mathbf{w}, \mathbf{v}}$ [21]. The formula in [3] amounts to replacing Euler characteristic of $T_{q}^{*} \mathfrak{M}_{\mathbf{w}, \mathbf{v}}$ by the $q_{2}$-equivariant Euler class of the tangent 
bundle to $T_{q}^{*} \mathfrak{M}_{\mathbf{w}, \mathbf{v}}$ so effectively to the integration over $\Pi T_{q_{2}} T_{q}^{*} \mathfrak{M}_{\mathbf{w}, \mathbf{v}}$. Here $\mathbf{w}: \Gamma_{0} \rightarrow \mathbb{Z}$ labels the components of the highest weight in the basis of fundamental weights, and $\mathbf{v}: \Gamma_{0} \rightarrow \mathbb{Z}$ labels the components of a positive root in the basis of simple roots which is added to the highest weight to get a weight at the level $\sum_{i \in \Gamma_{0}} \mathbf{v}_{i}$ in the Verma module.

5.2. Higher weight currents. Conjecturally, quiver $\mathrm{W}$-algebra is completely generated by the fundamental currents

$$
T_{i, x}=\mathrm{Y}_{i, x}+\ldots
$$

However, higher weight currents $T_{w, x}^{\mathbf{w}}$ can be defined where to each node $i$ we assign vector space $\mathbf{W}$ of dimension $\mathbf{w}$ and the character

$$
W_{i}=\sum_{\omega=1}^{\mathbf{w}_{i}} w_{i, \omega} \quad w_{i, \omega} \in \mathbb{C}^{\times}
$$

with the first term

$$
T_{w, x}^{\mathbf{w}}=: \prod_{i \in \Gamma_{0}} \prod_{\omega=1}^{\mathbf{w}_{i}} \mathrm{Y}_{i, w_{i, \omega} x}:+\ldots
$$

In the finite-dimensional and irreducible modules of higher weights can be found in the tensor product of the $i$-fundamental modules with weights $\mathbf{w}_{i}=1, \mathbf{w}_{j \neq i}=0$. In the not $q$ deformed case, usually the tensor product of fundamental modules decomposes into several irreducible components. For example, for $\mathfrak{s l}_{2}$ we have $\mathbb{C}^{2} \otimes \mathbb{C}^{2}=\mathbb{C}^{3} \oplus \mathbb{C}^{1}$. This does not hold after $q$-deformation. For generic weights $w$ the tensor product is irreducible.

5.3. Higher weight current in the $A_{1}$ example. In the example of $A_{1}$ quiver the higher weight current $T_{w, x}^{\mathbf{w}}$ with $\mathbf{w}_{1} \in \mathbb{Z}_{>0}$ for generic weights $\left(w_{1,1}, \ldots, w_{1, \mathbf{w}_{1}}\right)$ contains $2^{\mathbf{w}_{1}}$ terms [3] coming from the cohomologies of Nakajima's quiver variety which in this case are $\amalg_{\mathbf{v} \leq \mathbf{w}} T^{*} \mathbf{G r}(\mathbf{w}, \mathbf{v})$. This higher weight character current $T_{w, x}^{\mathbf{w}}$ is elementary to compute from the free-field formalism and normal ordering given the fundamental current $T_{1, x}$ in equation (4.1)

Consider the product

$$
T_{1, w_{1} x} T_{1, w_{2} x}=\left(\mathrm{Y}_{1, x w_{1}}+\mathrm{Y}_{1, q^{-1} w_{1} x}^{-1}\right)\left(\mathrm{Y}_{1, x w_{2}}+\mathrm{Y}_{1, q^{-1} w_{2} x}^{-1}\right)
$$

The normal ordering is computed using the commutator from (3.47)

$$
\left[y_{i, p}, y_{j,-p}\right] \stackrel{p>0}{=}-\frac{1}{p}\left(1-q_{1}^{p}\right)\left(1-q_{2}^{p}\right) \tilde{c}_{j i}^{[-p]}
$$

with the result

$$
\begin{aligned}
T_{1, w_{1} x} T_{1, w_{2} x}= & f\left(w_{2} / w_{1}\right)^{-1}\left(: \mathrm{Y}_{1, w_{1} x} \mathrm{Y}_{1, w_{2} x}:\right. \\
& +\mathcal{S}\left(w_{1} / w_{2}\right): \mathrm{Y}_{1, w_{1} x} \mathbf{Y}_{1, q^{-1} w_{2} x}^{-1}:+\mathcal{S}\left(w_{2} / w_{1}\right): \mathbf{Y}_{1, q^{-1} w_{1} x}^{-1} \mathbf{Y}_{1, w_{2} x}: \\
& \left.+: \mathbf{Y}_{1, q^{-1} w_{1} x}^{-1} \mathbf{Y}_{1, q^{-1} w_{2} x}^{-1}:\right)
\end{aligned}
$$

where the scalar prefactor

$$
f(w)=\exp \left(\sum_{p=1}^{\infty} \frac{1}{p} \frac{\left(1-q_{1}^{p}\right)\left(1-q_{2}^{p}\right)}{1+q^{p}} w^{p}\right)
$$


is in agreement with the function $f(w)$ generating the commutation relations for $W_{q_{1}, q_{2}}\left(A_{1}\right)$ current $T_{1}(x)$ in Shiraishi et al. [5] and the permutation factor $\mathcal{S}(u)$ is in agreement with formulae for higher $q q$-characters in [3]

$$
\mathcal{S}(w)=\frac{\left(1-q_{1} w\right)\left(1-q_{2} w\right)}{(1-q w)(1-w)}
$$

which comes from the equivariant Euler characteristic (or its K-theory version) of the fixed point in $\Pi T_{q_{2}} T_{q_{1}}^{*} \mathbb{P}^{1}$ where $T_{q_{1}}^{*} \mathbb{P}^{1}=\mathfrak{M}_{\mathbf{w}=2, \mathbf{v}=1}^{\text {Nak }}$. This relation leads to

$$
f\left(\frac{w_{2}}{w_{1}}\right) T_{1, w_{1} x} T_{1, w_{2} x}-f\left(\frac{w_{1}}{w_{2}}\right) T_{1, w_{1} x} T_{1, w_{2} x}=\frac{\left(1-q_{1}\right)\left(1-q_{2}\right)}{1-q}\left(\delta\left(q \frac{w_{1}}{w_{2}}\right)-\delta\left(q \frac{w_{2}}{w_{1}}\right)\right),
$$

which determines the algebraic relation for the modes $\left(T_{1,[p]}\right)_{p \in \mathbb{Z}}$. We remark $f(w) f(q w)=$ $\mathcal{S}(w)$.

The degree w current is similarly computed

$$
\begin{aligned}
T_{1, x}^{[\mathrm{w}]} & =: \mathrm{Y}_{1, w_{1} x} \mathrm{Y}_{1, w_{2} x} \cdots \mathrm{Y}_{1, w_{\mathrm{w}} x}:+\cdots \\
& =\sum_{I \cup J=\{1 \ldots \mathrm{w}\}} \prod_{i \in I, j \in J} \mathcal{S}\left(\frac{w_{i}}{w_{j}}\right): \prod_{i \in I} \mathrm{Y}_{1, w_{i} x} \prod_{j \in J} \mathrm{Y}_{1, q^{-1} w_{j} x}^{-1}:
\end{aligned}
$$

in agreement with [3]. The $\mathcal{S}$ factor becomes trivial in the limit $q_{2} \rightarrow 1$ and the ordinary formulae for $q$-character is recovered [2, 15, 16].

5.4. Degeneration and derivatives. By definition, vertex operator algebra involves expressions in fields and their derivatives. Hence we shall expect appearance of the derivatives when two vertex operators fuse.

So consider slightly more general situation of W-algebra currents with local structure

$$
\begin{aligned}
: Y_{i, x} Y_{i, u x}: & +\mathcal{S}(u): \frac{\mathrm{Y}_{i, u x}}{\mathrm{Y}_{i, q^{-1} x}}\left(\prod_{e: i \rightarrow j} \mathrm{Y}_{j, \mu_{e}^{-1} x} \prod_{e: j \rightarrow i} \mathrm{Y}_{j, \mu_{e} q^{-1} x}\right): \\
& +\mathcal{S}\left(u^{-1}\right): \frac{\mathrm{Y}_{i, x}}{\mathrm{Y}_{i, u q^{-1} x}}\left(\prod_{e: i \rightarrow j} \mathrm{Y}_{j, u \mu_{e}^{-1} x} \prod_{e: j \rightarrow i} \mathrm{Y}_{j, u \mu_{e} q^{-1} x}\right): \\
& +: \mathrm{Y}_{i, q^{-1} x}^{-1} \mathrm{Y}_{i, u q^{-1} x}^{-1}\left(\prod_{e: i \rightarrow j} \mathrm{Y}_{j, \mu_{e}^{-1} x} \mathrm{Y}_{j, u \mu_{e}^{-1} x} \prod_{e: j \rightarrow i} \mathrm{Y}_{j, \mu_{e} q^{-1} x} \mathrm{Y}_{j, u \mu_{e} q^{-1} x}\right):
\end{aligned}
$$

Taking the collision limit $u \rightarrow 1$, this yields a derivative term

$$
\begin{aligned}
\mathrm{Y}_{i, x}^{2}+ & : \frac{\mathrm{Y}_{i, x}}{\mathrm{Y}_{i, q^{-1} x}} \prod_{e: i \rightarrow j} \mathrm{Y}_{j, \mu_{e}^{-1} x} \prod_{e: j \rightarrow i} \mathrm{Y}_{j, \mu_{e} q^{-1} x} \\
& \times\left(\mathfrak{c}\left(q_{1}, q_{2}\right)-\frac{\left(1-q_{1}\right)\left(1-q_{2}\right)}{1-q} \frac{\partial}{\partial \log x} \log \left(\frac{\mathrm{Y}_{i, x} \mathrm{Y}_{i, q^{-1} x}}{\prod_{e: i \rightarrow j} \mathrm{Y}_{j, \mu_{e}^{-1} x} \prod_{e: j \rightarrow i} \mathrm{Y}_{j, \mu_{e} q^{-1} x}}\right)\right): \\
+ & : \mathrm{Y}_{i, q^{-1} x}^{-2}\left(\prod_{e: i \rightarrow j} \mathrm{Y}_{j, \mu_{e}^{-1} x} \prod_{e: j \rightarrow i} \mathrm{Y}_{j, \mu_{e} q^{-1} x}\right)^{2}:
\end{aligned}
$$


where the coefficient $\mathfrak{c}\left(q_{1}, q_{2}\right)$ is determined by

$$
\begin{aligned}
\mathfrak{c}\left(q_{1}, q_{2}\right) & =\lim _{u \rightarrow 1}\left(\mathcal{S}(u)+\mathcal{S}\left(u^{-1}\right)\right) \\
& =\frac{1-6 q_{1} q_{2}+q_{1}^{2} q_{2}^{2}+\left(1+q_{1} q_{2}\right)\left(q_{1}+q_{2}\right)}{\left(1-q_{1} q_{2}\right)^{2}} \stackrel{q_{1,2} \rightarrow 1}{\longrightarrow} 2 .
\end{aligned}
$$

We can consider more higher collision, which involves correspondingly higher derivatives.

5.5. Edge loop: $\widehat{A}_{0}$-example. Consider an example of a single node with a loop edge. This corresponds to $\mathcal{N}=2^{*}$ theory in 4 d. Let $\mathrm{n} \in \mathbb{Z}_{\geq 1}$ be the gauge group rank and $\mu \in \mathbb{C}^{\times}$ be the (multiplicative; exponentiated) adjoint mass. The Cartan matrix is (0) and the mass deformed Cartan matrix is

$$
c=1+q^{-1}-\mu^{-1}-q^{-1} \mu=\left(1-\mu^{-1}\right)\left(1-q^{-1} \mu\right)
$$

The quantum affinization of the respective algebra by Nakajima's quiver construction is $\mathbf{U}_{q, \mu}\left(\widehat{L g l}_{1}\right)$ [45 47] with $q$-character given by the sum over all partitions [2]. Here we consider $q_{2}$-deformation to recover $\mathrm{W}$-algebra of $\widehat{A}_{0}$-quiver.

We need the commutation relation for the oscillator (3.47)

$$
\left[y_{1, p}, y_{1, p^{\prime}}\right]=-\delta_{p+p^{\prime}, 0} \frac{1}{p} \frac{\left(1-q_{1}^{p}\right)\left(1-q_{2}^{p}\right)}{\left(1-\mu^{p}\right)\left(1-q^{p} \mu^{-p}\right)}
$$

Using this oscillator, we construct $W_{q_{1}, q_{2}}\left(\mathfrak{g}_{\Gamma}\right)$ algebra associated with the affine quiver $\Gamma=\widehat{A}_{0}$. In this case, the local pole cancellation structure is

$$
\mathrm{Y}_{1, x}+\mathcal{S}\left(\mu^{-1}\right): \mathrm{Y}_{1, q^{-1} x}^{-1} \mathrm{Y}_{1, \mu^{-1} x} \mathrm{Y}_{1, \mu q^{-1} x}:
$$

and the holomorphic current can be characterized by a single partition [2]

$$
\begin{aligned}
T_{1, x} & =\mathrm{Y}_{1, x}+\mathcal{S}\left(\mu^{-1}\right): \mathrm{Y}_{1, q^{-1} x}^{-1} \mathrm{Y}_{1, \mu^{-1} x} \mathrm{Y}_{1, \mu q^{-1} x}:+\cdots \\
& =\sum_{\lambda} \tilde{Z}_{\lambda}: \prod_{s \in \partial_{+} \lambda} \mathrm{Y}_{1, q x / \tilde{x}(s)} \prod_{s \in \partial_{-} \lambda} \mathrm{Y}_{1, x / \tilde{x}(s)}^{-1}:
\end{aligned}
$$

where $\partial_{+} \lambda$ and $\partial_{-} \lambda$ are the outer and inner boundary of the partition $\lambda$, and we define

$$
\tilde{x}(s)=\left(\mu^{-1} q\right)^{s_{1}-1} \mu^{s_{2}-1} q
$$

The combinatorial weight $\tilde{Z}_{\lambda}$ obeys

$$
\frac{\tilde{Z}_{\lambda^{\prime}}}{\tilde{Z}_{\lambda}}=-\left.\frac{\left(1-\mu^{-1} q_{1}\right)\left(1-\mu^{-1} q_{2}\right)}{\left(1-q_{1}\right)\left(1-q_{2}^{-1}\right)} \frac{\tilde{Y}_{q_{1} x} \tilde{Y}_{q_{2} x}}{\tilde{Y}_{q x}^{\prime} \tilde{Y}_{x}}\right|_{x=\tilde{x}_{k}}
$$

where $\lambda^{\prime}$ is the shifted partition $\lambda_{k} \rightarrow \lambda_{k}+1$, and we define the "dual" function $\tilde{Y}_{x}$

$$
\tilde{\mathrm{Y}}_{x}=\prod_{k=1} \frac{1-\tilde{x}_{k} / x}{1-\tilde{q}_{1} \tilde{x}_{k} / x}
$$

with the "dual" parameters

$$
\tilde{q}_{1}=\mu^{-1} q, \quad \tilde{q}_{2}=\mu, \quad \tilde{\mu}=q_{2}, \quad \tilde{x}_{k}=\tilde{x}\left(k, \lambda_{k}+1\right)
$$


Here $\tilde{Y}^{\prime}$ is evaluated with the shifted configuration $\lambda^{\prime}$. Although this dual function also has poles, such a singularity is cancelled in the following combination

$$
\tilde{\mathrm{Y}}_{x}+\frac{\left(1-\tilde{\mu}^{-1} \tilde{q}_{1}\right)\left(1-\tilde{\mu}^{-1} \tilde{q}_{2}\right)}{\left(1-\tilde{\mu}^{-1} \tilde{q}\right)\left(1-\tilde{Y}^{-1}\right)} \tilde{q}^{-1} \tilde{Y}_{\tilde{\mu}^{-1} x} \tilde{Y}_{\tilde{\mu} \tilde{q} x}
$$

This expression is equivalent to the original one (5.22) in particular for the rank one theory. Again, operator formalism of W-algebra is equivalent to $q_{2}$-deformation of Nakajima's construction [3].

5.6. W-algebra of hyperbolic quiver example. We consider examples of the hyperbolic quiver, where the determinant of the corresponding Cartan matrix is negative. The simplest example is the quiver having a single node with two loop edges:

$$
\subset \bullet \longmapsto \quad c=-(2)
$$

Let $\mu_{1,2} \in \mathbb{C}^{\times}$be the mass parameter associated with the edges, and the mass deformed Cartan matrix is given by

$$
c=1+q^{-1}-\mu_{1}^{-1}-\mu_{1} q^{-1}-\mu_{2}^{-1}-\mu_{2} q^{-1}
$$

Since the local pole cancellation occurs in the following combination

$$
\mathrm{Y}_{1, x}+\mathcal{S}\left(\mu_{1}^{-1}\right) \mathcal{S}\left(\mu_{2}^{-1}\right): \mathrm{Y}_{1, q^{-1} x}^{-1} \mathrm{Y}_{1, \mu_{1}^{-1} x} \mathrm{Y}_{1, \mu_{1} q^{-1} x} \mathrm{Y}_{1, \mu_{2}^{-1} x} \mathrm{Y}_{1, \mu_{2} q^{-1} x}:
$$

the first few terms of the holomorphic current are given by

$$
\begin{aligned}
& T_{1, x}=\mathrm{Y}_{1, x}+\mathcal{S}\left(\mu_{1}^{-1}\right) \mathcal{S}\left(\mu_{2}^{-1}\right): \mathrm{Y}_{1, q^{-1} x}^{-1} \mathrm{Y}_{1, \mu_{1}^{-1} x} \mathrm{Y}_{1, \mu_{1} q^{-1} x} \mathrm{Y}_{1, \mu_{2}^{-1} x} \mathrm{Y}_{1, \mu_{2} q^{-1} x}: \\
& +\left(\mathcal{S}\left(\mu_{1}^{-1}\right) \mathcal{S}\left(\mu_{2}^{-1}\right)\right)^{2}[ \\
& \mathcal{S}\left(\mu_{1}^{2} q^{-1}\right) \mathcal{S}\left(\mu_{1} \mu_{2}^{-1}\right) \mathcal{S}\left(\mu_{1} \mu_{2} q^{-1}\right): \frac{\mathrm{Y}_{1, \mu_{1}^{-2} x} \mathrm{Y}_{1, \mu_{1} q^{-1} x} \mathrm{Y}_{1, \mu_{1}^{-1} \mu_{2}^{-1} x} \mathrm{Y}_{1, \mu_{1}^{-1} \mu_{2} q^{-1} x} \mathrm{Y}_{1, \mu_{2}^{-1} x} \mathrm{Y}_{1, \mu_{2} q^{-1} x}}{\mathrm{Y}_{1, \mu_{1}^{-1} q^{-1} x}}: \\
& +\mathcal{S}\left(\mu_{1}^{-2} q\right) \mathcal{S}\left(\mu_{1}^{-1} \mu_{2}^{-1} q\right) \mathcal{S}\left(\mu_{1}^{-1} \mu_{2}\right): \frac{\mathrm{Y}_{1, \mu_{1}^{-1} x} \mathrm{Y}_{1, \mu_{1}^{2} q^{-2} x} \mathrm{Y}_{1, \mu_{1} \mu_{2}^{-1} x} \mathrm{Y}_{1, \mu_{1} \mu_{2} q^{-1} x} \mathrm{Y}_{1, \mu_{2}^{-1} x} \mathrm{Y}_{1, \mu_{2} q^{-1} x}}{\mathrm{Y}_{1, \mu_{1} q^{-2} x}}: \\
& +(1 \leftrightarrow 2)]+\cdots
\end{aligned}
$$

We can see a cancellation of factors, which is similar to $\hat{A}_{0}$ theory, and thus there is no colliding term, e.g. $\mathrm{Y}_{1, *}^{2}$, in a numerator.

Next example is a rank two quiver with three arrows:

$$
\longrightarrow \quad c=\left(\begin{array}{cc}
2 & -3 \\
-3 & 2
\end{array}\right)
$$

Let us assign three mass parameters $\mu_{1,2,3}$ to the arrows, and then the local cancellation is

$$
\mathrm{Y}_{1, x}+: \frac{\mathrm{Y}_{2, \mu_{1}^{-1} x} \mathrm{Y}_{2, \mu_{2}^{-1} x} \mathrm{Y}_{2, \mu_{3}^{-1} x}}{\mathrm{Y}_{1, q^{-1} x}}: \quad \mathrm{Y}_{2, x}+: \frac{\mathrm{Y}_{1, \mu_{1} q^{-1} x} \mathrm{Y}_{1, \mu_{2} q^{-1} x} \mathrm{Y}_{1, \mu_{3} q^{-1} x}}{\mathrm{Y}_{2, q^{-1} x}}:
$$


The holomorphic current becomes

$$
\begin{aligned}
T_{1, x}= & \mathrm{Y}_{1, x}+ \\
+ & : \frac{\mathrm{Y}_{2, \mu_{1}^{-1} x} \mathrm{Y}_{2, \mu_{2}^{-1} x} \mathrm{Y}_{2, \mu_{3}^{-1} x}}{\mathrm{Y}_{1, q^{-1} x}}: \\
& +\mathcal{S}\left(\mu_{1} \mu_{2}^{-2}\right) \mathcal{S}\left(\mu_{1} \mu_{3}^{-1}\right): \frac{\mathrm{Y}_{1, \mu_{1}^{-1} \mu_{2} q^{-1}} \mathrm{Y}_{1, \mu_{1}^{-1} \mu_{3} q^{-1}} \mathrm{Y}_{2, \mu_{2}^{-1} x} \mathrm{Y}_{2, \mu_{3}^{-1} x}}{\mathrm{Y}_{2, \mu_{1}^{-1} q^{-1} x}}: \\
& +\mathcal{S}\left(\mu_{2} \mu_{1}^{-2}\right) \mathcal{S}\left(\mu_{2} \mu_{3}^{-1}\right): \frac{\mathrm{Y}_{1, \mu_{2}^{-1} \mu_{1} q^{-1}} \mathrm{Y}_{1, \mu_{2}^{-1} \mu_{3} q^{-1} \mathrm{Y}_{2, \mu_{1}^{-1} x} \mathrm{Y}_{2, \mu_{3}^{-1} x}}}{\mathrm{Y}_{2, \mu_{2}^{-1} q^{-1} x}}: \\
& \left.+\mathcal{S}\left(\mu_{3} \mu_{1}^{-2}\right) \mathcal{S}\left(\mu_{3} \mu_{1}^{-1}\right): \frac{\mathrm{Y}_{1, \mu_{3}^{-1} \mu_{1} q^{-1}} \mathrm{Y}_{1, \mu_{3}^{-1} \mu_{2} q^{-1} \mathrm{Y}_{2, \mu_{1}^{-1} x} \mathrm{Y}_{2, \mu_{2}^{-1} x}}}{\mathrm{Y}_{2, \mu_{3}^{-1} q^{-1} x}}\right] \\
& +\cdots
\end{aligned}
$$

The other current $T_{2, x}$ is obtained in the same way. Similarly it is expected that there is no collision term in these holomorphic currents.

\section{Applications}

6.1. Toda scaling limit. In the scaling limit $q_{1} \rightarrow 1, q_{2} \rightarrow 1, \mathfrak{q}_{i} \rightarrow 1$, and $\log _{q_{2}} q_{1}, \log _{q_{2}} \mathfrak{q}_{i}$ are finite, the free field commutation relations (3.33) (3.34) turn into

$$
\begin{aligned}
{\left[s_{i, p}, s_{j, p^{\prime}}\right] } & =-\delta_{p+p^{\prime}, 0} \frac{1}{p} \beta c_{j i}^{[0]}, \quad p>0 \\
{\left[\tilde{s}_{i, 0}, s_{j, p}\right] } & =-\beta \delta_{0, p} c_{j i}^{[0]}
\end{aligned}
$$

This limit was studied in details in section 4.1 of [1]. In terms of the parameter $b^{2}=-\beta$ the vertex operator (3.31) can be written as

$$
S_{i}(x)=: e^{b \phi_{i}(x)}:
$$

where $\phi_{i}(x)$ is the free boson that takes value in the Cartan part of $\mathfrak{g}_{\Gamma}$ with canonical commutation relations defined by the bilinear form with matrix $\left(c_{i j}\right)$ in the basis of simple roots. Hence $S_{i}(x)$ are vertex primary operators of Kac-Moody $\mathfrak{g}_{\Gamma}$-Toda field theory on punctured disc $\mathbb{C}_{x}^{\times}$. In the same scaling limit we find from (3.48) that the field $y_{i}(x)=$ $-\epsilon_{2} b \partial \phi_{j}(x) \tilde{c}_{j i}$ is also primary.

For example, in the $\epsilon_{2}$-expansion of $T_{1}(x)$ for $A_{1}$-quiver

$$
T_{1}(x)=: e^{y(x)}:+: e^{-y\left(x q^{-1}\right)}:=2+\frac{1}{4} \epsilon^{2} b^{2}\left((\partial \phi)^{2}-\left(b+b^{-1}\right) \partial^{2} \phi\right)+\ldots
$$

we find the stress-energy Virasoro current of the free field $\phi$ with background charge and the central charge

$$
c=1+6\left(b+b^{-1}\right)^{2}
$$

6.2. Affine type. If $\mathfrak{g}_{\Gamma}$ is of affine type, the $\mathfrak{g}_{\Gamma^{-}}$-Toda is affine Toda. For example, the scaling limit of the $\mathrm{W}$-algebra defined by the quiver

$$
\longrightarrow c=\left(\begin{array}{cc}
2 & -2 \\
-2 & 2
\end{array}\right)
$$

with $\mathfrak{g}_{\Gamma}=A_{1}^{(1)}$ describes quantum $\sin (\mathrm{h})$-Gordon theory on punctured disc $\mathbb{C}_{x}^{\times}$. 
6.3. Nahm transform. The $\mathfrak{g}_{\Gamma^{-}}$Toda theory specializes to the finite Toda if $\mathfrak{g}_{\Gamma}$ is of finite type. For $\mathfrak{s l}_{r}$-quiver with $n$ colors at each node the $\mathfrak{s l}_{r}$-Toda is Nahm dual to the $\mathfrak{s l}_{n}$-Toda proposed in [27, 28].

\section{REFERENCES}

[1] E. Frenkel and N. Reshetikhin, "Deformations of $\mathcal{W}$-algebras associated to simple Lie algebras," Comm. Math. Phys. 197 (1998) 1-32, q-alg/9708006 [math.QA].

[2] N. Nekrasov, V. Pestun, and S. Shatashvili, "Quantum geometry and quiver gauge theories," Commun. Math. Phys. 357 (2018) 519-567, arXiv:1312.6689 [hep-th].

[3] N. Nekrasov, "BPS/CFT correspondence: non-perturbative Dyson-Schwinger equations and qq-characters," JHEP 1603 (2016) 181, arXiv:1512.05388 [hep-th].

[4] A. Marshakov and N. Nekrasov, "Extended Seiberg-Witten Theory and Integrable Hierarchy," JHEP 0701 (2007) 104, hep-th/0612019.

[5] J. Shiraishi, H. Kubo, H. Awata, and S. Odake, "A Quantum deformation of the Virasoro algebra and the Macdonald symmetric functions," Lett. Math. Phys. 38 (1996) 33-51, q-alg/9507034.

[6] E. Frenkel and N. Reshetikhin, "Quantum affine algebras and deformations of the Virasoro and W-algebras," Comm. Math. Phys. 178 (1996) 237-264, q-alg/9505025.

[7] N. Nekrasov and V. Pestun, "Seiberg-Witten geometry of four dimensional $N=2$

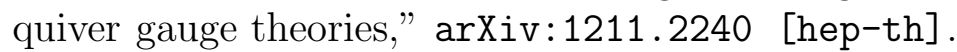

[8] B. Feigin and E. Frenkel, "Affine Kac-Moody algebras at the critical level and Gelfand-Dikii algebras," Int. J. Mod. Phys. A7 (1992) 197-215.

[9] E. Frenkel and D. Hernandez, "Langlands duality for finite-dimensional representations of quantum affine algebras," Lett. Math. Phys. 96 (2011) 217-261, arXiv:0902.0447 [math.QA]

[10] A. Beilinson and V. Drinfeld, "Quantization of Hitchin's integrable system and Hecke eigensheaves,". http://www .math.uchicago.edu/ mitya/langlands/QuantizationHitchin.pdf.

[11] A. Kapustin and E. Witten, "Electric-Magnetic Duality And The Geometric Langlands Program," Commun. Num. Theor. Phys. 1 (2007) 1-236, arXiv:hep-th/0604151 [hep-th].

[12] N. Nekrasov and E. Witten, "The Omega Deformation, Branes, Integrability, and Liouville Theory," JHEP 1009 (2010) 092, 1002.0888.

[13] E. Frenkel, "Lectures on the Langlands program and conformal field theory," in Frontiers in Number Theory, Physics, and Geometry II, pp. 387-533. Springer, 2007. arXiv:hep-th/0512172 [hep-th].

[14] J. C. Hurtubise and E. Markman, "Elliptic Sklyanin integrable systems for arbitrary reductive groups," Adv. Theor. Math. Phys. 6 (2002) 873-978, math/0203031.

[15] E. Frenkel and N. Reshetikhin, "The $q$-characters of representations of quantum affine algebras and deformations of $\mathcal{W}$-algebras," in Recent Developments in Quantum Affine Algebras and Related Topics, vol. 248 of Contemp. Math., pp. 163-205. Amer. Math. Soc., 1999. math/9810055 [math.QA].

[16] E. Frenkel and D. Hernandez, "Baxter's relations and spectra of quantum integrable models," Duke Math. J. 164 no. 12, (2015) 2407-2460, arXiv:1308.3444 [math.QA].

[17] H. Nakajima, "Quiver varieties and finite-dimensional representations of quantum affine algebras," J. Amer. Math. Soc. 14 (2001) 145-238, math/9912158. 
[18] C. M. Ringel, "Hall algebras and quantum groups," Invent. Math. 101 (1990) 583-591.

[19] G. Lusztig, "Quivers, perverse sheaves, and quantized enveloping algebras," J. Amer. Math. Soc. 4 (1991) 365-421.

[20] V. Ginzburg and É. Vasserot, "Langlands reciprocity for affine quantum groups of type $A_{n}$, "Internat. Math. Res. Notices (1993) 67-85.

[21] H. Nakajima, "Quiver varieties and $t$-analogs of $q$-characters of quantum affine algebras," Ann. of Math. 160 (2004) 1057-1097, math/0105173.

[22] M. Kontsevich and Y. Soibelman, "Cohomological Hall algebra, exponential Hodge structures and motivic Donaldson-Thomas invariants,"

Commun. Num. Theor. Phys. 5 (2011) 231-352, arXiv:1006.2706 [math.AG].

[23] B.-Y. Hou and W.-L. Yang, "A $\hbar$-deformation of the $W_{N}$ algebra and its vertex operators," J. Phys. A30 (1997) 6131-6145, hep-th/9701101.

[24] A. Iqbal, C. Kozçaz, and S.-T. Yau, "Elliptic Virasoro Conformal Blocks," arXiv:1511.00458 [hep-th].

[25] F. Nieri, "An elliptic Virasoro symmetry in 6d," Lett. Math. Phys. 107 (2017) 2147-2187, arXiv:1511.00574 [hep-th].

[26] E. Witten, "Solutions of four-dimensional field theories via M theory," Nucl. Phys. B500 (1997) 3-42, hep-th/9703166.

[27] L. F. Alday, D. Gaiotto, and Y. Tachikawa, "Liouville Correlation Functions from Four-dimensional Gauge Theories," Lett. Math. Phys. 91 (2010) 167-197, arXiv:0906.3219 [hep-th].

[28] N. Wyllard, " $A_{N-1}$ conformal Toda field theory correlation functions from conformal $\mathcal{N}=2 S U(N)$ quiver gauge theories," JHEP 0911 (2009) 002, arXiv:0907.2189 [hep-th]

[29] A. Braverman, M. Finkelberg, and H. Nakajima, "Instanton moduli spaces and W-algebras," Astérisque 385 (2016), arXiv:1406.2381 [math.QA].

[30] A. Iqbal, C. Kozçaz, and C. Vafa, "The Refined topological vertex," JHEP 0910 (2009) 069, hep-th/0701156.

[31] H. Awata and H. Kanno, "Refined BPS state counting from Nekrasov's formula and Macdonald functions," Int. J. Mod. Phys. A24 (2009) 2253-2306, arXiv:0805.0191 [hep-th]

[32] N. Nekrasov and A. Okounkov, "Membranes and Sheaves," Alg. Geom. 3 (2016) 320-369, arXiv:1404.2323 [math.AG].

[33] L. Bao, E. Pomoni, M. Taki, and F. Yagi, "M5-Branes, Toric Diagrams and Gauge Theory Duality," JHEP 1204 (2012) 105, arXiv: 1112.5228 [hep-th].

[34] M. Aganagic and N. Haouzi, "ADE Little String Theory on a Riemann Surface (and Triality)," arXiv:1506.04183 [hep-th].

[35] S. Katz, P. Mayr, and C. Vafa, "Mirror symmetry and exact solution of 4-D $N=2$ gauge theories: 1.," Adv. Theor. Math. Phys. 1 (1998) 53-114, hep-th/9706110.

[36] B. Eynard, "All orders asymptotic expansion of large partitions," J. Stat. Mech. 0807 (2008) P07023, arXiv:0804.0381 [math-ph].

[37] P. Sulkowski, "Matrix models for $\beta$-ensembles from Nekrasov partition functions," JHEP 1004 (2010) 063, 0912.5476.

[38] A. Nedelin and M. Zabzine, " $q$-Virasoro constraints in matrix models," JHEP 1703 (2017) 98, arXiv:1511.03471 [hep-th]. 
[39] A. Mironov, A. Morozov, and Y. Zenkevich, "On elementary proof of AGT relations from six dimensions," Phys. Lett. B756 (2016) 208-211, arXiv:1512.06701 [hep-th].

[40] I. K. Kostov, "Gauge invariant matrix model for the A-D-E closed strings," Phys. Lett. B297 (1992) 74-81, hep-th/9208053.

[41] J.-E. Bourgine, Y. Mastuo, and H. Zhang, "Holomorphic field realization of $\mathrm{SH}^{c}$ and quantum geometry of quiver gauge theories," JHEP 1604 (2016) 167, arXiv:1512.02492 [hep-th].

[42] H. Nakajima, "Instantons on ALE spaces, quiver varieties, and Kac-Moody algebras," Duke Math. J. 76 (1994) 365-416.

[43] V. Ginzburg, M. Kapranov, and E. Vasserot, "Langlands reciprocity for algebraic surfaces," Math. Res. Lett. 2 (1995) 147-160, q-alg/9502013.

[44] M. Kapranov and E. Vasserot, "Kleinian singularities, derived categories and Hall algebras," Math. Ann. 316 (2000) 565-576, math/9812016 [math. AG].

[45] O. Schiffmann and E. Vasserot, "The elliptic Hall algebra and the equivariant K-theory of the Hilbert scheme of $\mathbb{A}^{2}$," Duke Math. J. 162 (2013) 279-366, arXiv:0905.2555 [math.QA].

[46] M. Varagnolo and E. Vasserot, "On the $K$-theory of the cyclic quiver variety," Internat. Math. Res. Notices (1999) 1005-1028, math/9902091.

[47] O. Schiffmann and E. Vasserot, "Cherednik algebras, W-algebras and the equivariant cohomology of the moduli space of instantons on $\mathbb{A}^{2}$,"

Publ. Math. IHES 118 (2013) 213-342, arXiv:1202.2756 [math.QA].

[48] D. Maulik and A. Okounkov, "Quantum Groups and Quantum Cohomology," arXiv:1211.1287 [math.AG].

[49] M. R. Douglas and G. W. Moore, "D-branes, Quivers, and ALE Instantons," hep-th/9603167.

[50] N. A. Nekrasov, "Seiberg-Witten prepotential from instanton counting," Adv. Theor. Math. Phys. 7 (2004) 831-864, hep-th/0206161.

[51] G. W. Moore, N. Nekrasov, and S. Shatashvili, "Integrating over Higgs branches," Commun. Math. Phys. 209 (2000) 97-121, hep-th/9712241.

[52] H. Nakajima, Lectures on Hilbert Schemes of Points on Surfaces, vol. 18 of University Lecture Series. AMS, 1999.

Taro Kimura, Keio University, Japan

Vasily Pestun, IHeS, France 